\title{
Capturing the Transient Microstructure of a Physically Assembled Gel Subjected to Temperature and Large Deformation
}

\author{
Rosa Maria Badani Prado, ${ }^{\dagger}$ Satish Mishra, ${ }^{\dagger}$ Wesley R. Burghardt, ${ }^{\ddagger}$ and Santanu \\ Kundu $u^{* \dagger}$ \\ $\dagger$ Dave C. Swalm School of Chemical Engineering, Mississippi State University, MS State, \\ MS 39762. \\ $\ddagger$ Department of Chemical Engineering, Northwestern University, Evanston, IL 60208 \\ E-mail: santanukundu@che.msstate.edu
}

\begin{abstract}
The microstructure of physically assembled gels depends on mechanical loading and environmental stimuli such as temperature. Here, we report the real-time change in the structure of physically assembled triblock copolymer gels that consist of $10 \mathrm{wt} \%$ and $20 \mathrm{wt} \%$ of poly(styrene)-poly(isoprene)-poly(styrene) [PS-PI-PS] triblock copolymer in mineral oil (i) during the gelation process with decreasing temperature, (ii) subjected to large oscillatory deformation, and (iii) during the stress-relaxation process after the application of a step-strain. The presence of loosely bounded PS-aggregates at temperatures higher than the rheometrically determined gelation temperature $\left(T_{\text {gel }}\right)$ captures the progressive gelation process spanning over a broad temperature range. However, the microstructure fully develops at temperatures sufficiently lower than $T_{\text {gel }}$, and the storage modulus $\left(G^{\prime}\right)$ also reaches a plateau at those temperatures. The microstructure orients in the stretching direction with the applied strain. In an oscillation strain cycle,
\end{abstract}


such oriented structure has been observed at low-strain. But, at large-strain, the oriented structure splits, and only a fraction of midblock participates in load-bearing. This has been attributed to the endblock pullout from the aggregates, likely caused by the strain localization in the samples. Both microstructure recovery and time-dependent moduli during the stress-relaxation process after the application of a step-strain can be captured using a stretched-exponential model. However, the microstructure recovery time has been found to be two orders of magnitude slower than the stress-relaxation time at room temperature, indicating a complex nature of relaxation process involving midblock relaxation, endblock pullout and reassociation process. Due to their viscoelastic nature, these gels' mechanical responses are sensitive to strain, temperature, and rate of deformation. Therefore, insights into the microstructural information as a function of these parameters will assist these gels' real-life applications and design new gels with improved properties.

\section{Introduction}

ABA triblock copolymer gels in B-selective solvents are a class of gels that have garnered significant research interests over the last few decades. ${ }^{1-8}$ These gels have been investigated for their potential applications in various areas ranging from biomedical fields as wound dressing, ${ }^{9}$ protein carriers, ${ }^{10}$ and drug delivery, ${ }^{11,12}$ to ballistics, ${ }^{13}$ microfluidic devices, ${ }^{14}$ and soft robotics. ${ }^{15,16}$ These gels are significantly different than chemically crosslinked gels, ${ }^{16,17}$ and the underlying principle of gelation in these gels is the strong temperature-dependent solubility of the A-blocks (endblock) in the chosen solvent, in comparison to the very little change of solubility of the B-blocks (midblock) with temperature. Specifically, at higher temperatures, both A- and B-blocks are soluble in the solvent, however, the A-block's solubility becomes poor with the decrease of temperature. This causes the aggregation of a few A-blocks at low temperatures, forming physically associated crosslinks (aggregates) bridged by B-blocks. The B-blocks remain soluble in the solvent and act as load-bearing chains. This process leads 
to the formation of a physically assembled triblock copolymer gel. The gels investigated in this study consist of poly(styrene)-poly(isoprene)-poly(styrene) [PS-PI-PS] in mineral oil. Here, the PS-blocks form the aggregates, and these are connected by load-bearing bridges of PI-blocks. ${ }^{1,2,4,6,7}$ Due to the temperature-dependent solubility of A- and B-blocks, these gels are thermoreversible, and become a polymer solution ( $\mathrm{sol}$ ) at higher temperatures and gel again at lower temperatures.

Previously, there has been a significant effort to understand the effect of polymer volume fraction $(\phi),{ }^{4,7,18}$ temperature, ${ }^{4,18}$ block lengths, ${ }^{7,13,19-21}$ endblock homopolymer addition, ${ }^{5}$ and solvent ${ }^{22}$ on the physical properties of triblock copolymer gels, such as gelation behavior, critical gelation concentration, stress-relaxation, moduli, and rate-dependent responses. However, most of these properties were realized through macroscopic experimental observations, and the relationships between their mechanical properties and the microstructure are not well established.

Small-angle neutron/X-ray (SANS/SAXS) scattering has been used to understand the change in ABA gel microstructure while varying the $\phi,{ }^{1,4}$ endblock and midblock lengths,,${ }^{4,7}$ and temperature, ${ }^{4,18}$ but mostly at the static condition. In those studies, structural information obtained from the scattering data at the static condition has been used to explain the gel responses subjected to mechanical deformation, leaving a gap between the theoretical understanding, prediction, and the experimental observations. For example, the characteristic time-scale for stress-relaxation in these gels determined from rheological experiments has been linked to the endblocks pullout from the aggregates and their reassociation with other aggregates. ${ }^{2,4,6,7,18,23-25}$ However, no information is available regarding the time scale associated with complete microstructure recovery. Therefore, real-time observation of the change in gel microstructure subjected to a mechanical deformation can provide further understanding regarding the microstructure-dependent mechanical properties of these gels, including structural reorganization during the relaxation process.

Such investigation requires real-time data collection using SAXS/SANS techniques while 
the gel is subjected to the mechanical loading. However, these experiments possess two main challenges. The first challenge is the requirement of a fast data collection necessary to capture the microstructure at any particular stress or strain value. This can be overcome by using a high flux beam typical to synchrotron. The second challenge is the availability of an instrument that allows the data collection along the appropriate plane. There are many reports in the literature to elucidate the real-time microstructural change during processing, and subjected to flow, mechanical loading, and magnetic field on polymers, ${ }^{26-32}$ complex fluids, ${ }^{33,34}$ protein gels, ${ }^{35}$ and wormlike micelles. ${ }^{36,37}$ In these studies, interesting microstructure development such as shear-rate dependent orientation development has been reported. Such investigations for ABA gels are limited, as the previous investigations on acrylic gels formed by poly(methyl methacrylate)-poly(n-butyl acrylate)-poly(methyl methacrylate) [PMMA-PnBA-PMMA] in 2-ethyl-1-hexanol and n-butanol capture the microstructure only at the static condition. ${ }^{7,22,38}$

For high modulus polymers and elastomers, it is possible to capture the real-time microstructural change as a function of uniaxial strain typically achieved in tensile testing. ${ }^{29,39}$ However, there are significant challenges to capture the real-time microstructure data during the tensile test of physically assembled gels. Particularly, because of their lower modulus, it is difficult to hold these samples in a tensile testing instrument. Also, the samples can undergo creep during the tests. ${ }^{6,7}$ Alternatively, the change in the gel microstructure subjected to mechanical deformation can be investigated in a shear-stage, where the scattering data collection is in the $\mathrm{x}-\mathrm{y}$ plane while the beam is in the $\mathrm{z}$-direction. Here " $\mathrm{x}$ " represents the flow/stretch direction $(v)$, " $\mathrm{y}$ " denotes velocity gradient $(\nabla v)$, and " $\mathrm{z}$ " denotes the vorticity direction $(v \times \nabla v)$. Using this setup, microstructural information in the stretch, or flow direction, and orthogonal to that, i.e., in the gradient direction can be collected. Our experimental setup is similar to that has been recently utilized to investigate the structural change in associative protein gels subjected to thermal load, oscillatory deformation, and during stress-relaxation. ${ }^{35}$ 
The present work focuses on real-time investigation of microstructural change in physi-

cally assembled PS-PI-Ps gels in mineral oil as a function of temperature, oscillatory strain, and during stress-relaxation. We have explored such transient microstructure in gels consisting of $10 \mathrm{wt} \%$ and $20 \mathrm{wt} \%$ of PS-PI-PS triblock copolymer, referred to as 10\%-Gel and 20\%-Gel, respectively. The PI-block molecular weight is higher than its entanglement molecular weight $(\approx 6.4 \mathrm{~kg} / \mathrm{mol})$, leading to a slight entanglement of PI-blocks in $20 \%$-Gel. We have used both the RheoSAXS and shear-rheology techniques in parallel to relate the microstructural change with the applied load since our RheoSAXS setup cannot measure the stress. ${ }^{30,35}$ The RheoSAXS data was collected in the $\mathrm{x}-\mathrm{y}$ plane, which provided a unique insight regarding the gel microstructure, particularly during large amplitude oscillatory strain (LAOS), where the structure changes reversibly in a strain-cycle.

\section{Experimental Methods}

Materials. Kraton D1164 is a poly(styrene)-poly(isoprene)-poly(styrene) [PS-PI-PS] triblock copolymer, kindly provided by Kraton Inc. Klearol white mineral oil (kindly provided by Sonneborn Inc.) was the solvent used for the gel preparation. ${ }^{6-8}$ The number-average $\left(M_{n}\right)$ and weight-average $\left(M_{w}\right)$ molecular weight of D1164 polymer are $\approx 112 \mathrm{~kg} / \mathrm{mol}$ and $\approx 125 \mathrm{~kg} / \mathrm{mol}$, respectively, estimated based on the polystyrene standard. ${ }^{6-8}$ The polymer has $29 \mathrm{wt} \%$ of PS and $71 \mathrm{wt} \%$ of PI, as indicated by the manufacturer. The estimated $M_{n}$ of the PS-block is $\approx 16.2 \mathrm{~kg} / \mathrm{mol}$, and that of the PI-block is $\approx 79.4 \mathrm{~kg} / \mathrm{mol}$.

Sample preparation. The samples were prepared by adding the desired amount of D1164 polymer in white mineral oil and mixing at $120^{\circ} \mathrm{C}$ by using a magnetic stirrer at $320 \mathrm{rpm}$. The polymer solutions were placed in a convective oven at $120{ }^{\circ} \mathrm{C}$ for $6 \mathrm{hrs}$, allowing the bubbles to escape, leading to clear solutions. Gels were obtained by cooling these solutions. We have considered two gels with $10 \mathrm{wt} \%$ and $20 \mathrm{wt} \%$ concentrations of D1164 polymer, corresponding to the polymer volume fractions $(\phi)$ of 0.089 and $0.181,{ }^{6,7}$ 


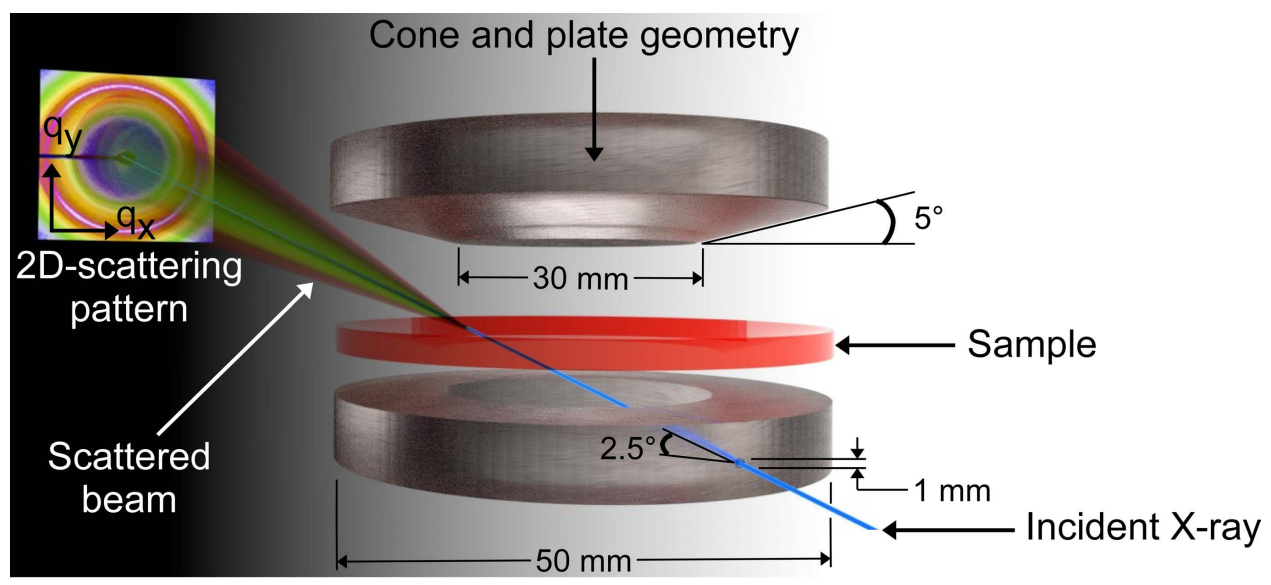

Figure 1: Schematic of the RheoSAXS setup with relevant details. The beam-path leading to data collection in the $\mathrm{x}-\mathrm{y}$ plane is also presented.

respectively. These gels are referred to as $10 \%$-Gel and $20 \%$-Gel throughout this article. Table S1 summarizes the polymer weight and volume fractions and the PS and PI weight fractions in each gel.

Shear-rheology experiments. For the shear-rheology experiments, polymer solution was poured in a rectangular mold of $2 \mathrm{~mm}$ thickness and was allowed to cool for $\approx 30 \mathrm{~min}$ through natural cooling, obtaining gel sheets. Square-shaped specimens $(30 \mathrm{~mm} \times 30 \mathrm{~mm})$ were cut from the gel sheets for individual rheology experiments. A TA Instruments Discovery HR-2 hybrid rheometer equipped with a Peltier plate was used. The experiments were conducted using a $25 \mathrm{~mm}$ parallel plate geometry with a gap of $1 \mathrm{~mm}$. 240 grit adhesivebacked silicon carbide sandpaper (Allied High Tech Products Inc.) was attached to the top and bottom plates to avoid possible slippage of samples on the rheometer plates. These experiments are referred to as shear-rheology experiments in the present article to distinguish them from the RheoSAXS experiments, which were performed in the synchrotron beamline as described below.

RheoSAXS experiments. The rheology experiments in conjunction with the smallangle X-ray scattering (RheoSAXS) experiments were conducted for the $10 \%$ - and 20\%-Gels on the DND-CAT beamline of the Advanced Photon Source at Argonne National Lab (ANL, 
Chicago, IL). The X-ray have a wavelength of $0.7293 \AA$ (energy=17 keV). A custom-built shear-stage equipped with an annular cone and plate geometry was used (Figure 1). ${ }^{30,35}$ The top geometry (cone) had a cone angle of $5^{\circ}$. The annular bottom plate had an inner diameter of $30 \mathrm{~mm}$ and an outer diameter of $50 \mathrm{~mm}$. A $1 \mathrm{~mm}$ diameter pinhole (beam path) drilled through the bottom plate of the shear-stage at an angle of $2.5^{\circ}$, allowed the X-ray beam to pass through the sample (Figure 1). This bottom plate was equipped with a thermocouple. The samples were loaded in the sol state at $\approx 80{ }^{\circ} \mathrm{C}$ and $100{ }^{\circ} \mathrm{C}$ for the $10 \%$ - and $20 \%$-Gel, respectively. For the experiments at room temperature, the samples were cooled down to room temperature $\left(\approx 27^{\circ} \mathrm{C}\right)$ by blowing air on the samples for $\approx 30$ minutes to ensure their thermal equilibrium. The shear-stage was then tilted by $2.5^{\circ}$ from its horizontal axis to align the beam-path for capturing the scattering data at the flow-vorticity plane. ${ }^{30,35,40}$ For the experiments where the scattering data were collected as a function of temperature, the samples were loaded in liquid state and the stage was tilted before cooling the sample. In these cases, the sample was allowed to cool naturally without blowing air onto the sample. However, for the experiments performed at higher temperatures than room temperature, like the stress-relaxation experiments at $40{ }^{\circ} \mathrm{C}$, the samples were brought down to experimental temperature by blowing air. A minimum waiting time of 15 min was allowed at the experimental temperature to attain thermal and structural equilibrium. Unless there was a macroscopic fracture, the same sample was used for multiple rheology experiments. However, after each experiment, the samples were heated to the sol state to erase the strain history. If the sample fractured during the test, a fresh sample was loaded using the same procedure discussed above.

The scattering data were collected for $10 \%$ - and $20 \%$-Gels in three sets of experiments viz. (1) temperature sweep without any applied strain as the gelation of sample taking place, (2) oscillatory shear experiments with strain amplitudes $\left(\gamma_{a m p}\right)$ of 0.1 and 1 , using a frequency $(\omega)$ of $1 \mathrm{rad} / \mathrm{s}$ at different temperatures, and (3) stress-relaxation experiments at different temperatures after the application of a step shear strain $\left(\gamma_{\text {step }}\right)$ of 0.3 . The two-dimensional 
(2D) scattering patterns were collected using a CCD detector. Igor Pro Nika package was used for data reduction from the 2D-scattering profiles to 1D intensity $(I(q))$ versus scattering vector $(q)$ plots. ${ }^{41}$ A custom-built NCNR macro was used to fit the data with a polydispersed core hard-sphere model and its modified form. ${ }^{42}$ Custom-built programs in MATLAB were also implemented to generate the azimuthal scans over a particular $q$-range and to estimate anisotropy. ${ }^{30,35}$

\section{Results and Discussion}

\section{Effect of Temperature on the Gel Microstructure}

The gelation temperature $\left(T_{g e l}\right)$ and moduli of the gels were captured by using shear-rheology experiments performed with an oscillation frequency $(\omega)$ of $1 \mathrm{rad} / \mathrm{s}$ and strain amplitude $\left(\gamma_{a m p}\right)$ of 0.01 over the temperature $(T)$ range of $80-20{ }^{\circ} \mathrm{C}$ and of $100-20{ }^{\circ} \mathrm{C}$ for the $10 \%$ - and the 20\%-Gels, respectively. Correspondingly, to capture the evolution of the gel microstructure with decreasing temperature, RheoSAXS experiments were performed over $\approx 80$ to $27^{\circ} \mathrm{C}$ for the $10 \%$-Gel, and from $\approx 120$ to $27{ }^{\circ} \mathrm{C}$ for the $20 \%$-Gel. Note that the RheoSAXS setup cannot measure the stress, therefore, the change in microstructure observed in the RheoSAXS experiments has been related to the data obtained from the shear-rheology experiments.

Figure 2A displays the evolution of storage $\left(G^{\prime}\right)$ and loss $\left(G^{\prime \prime}\right)$ moduli for $10 \%$ - and 20\%Gels as a function of temperature. At high temperature, both gels behave like a viscous liquid (sol-state) as $G^{\prime \prime}$ is higher than $G^{\prime}$. An increase in $G^{\prime}$ and $G^{\prime \prime}$ with the decrease of temperature indicates the formation of a network structure in these samples. At further lower temperature, a crossover of $G^{\prime}$ and $G^{\prime \prime}$ is observed, which can be identified as the gelation temperature $\left(T_{\text {gel }}\right)$ corresponding to $\omega=1 \mathrm{rad} / \mathrm{s}$. The $T_{\text {gel }}$ has been found to be $\approx 63{ }^{\circ} \mathrm{C}$ for $10 \%$-Gel and $\approx 85{ }^{\circ} \mathrm{C}$ for $20 \%$-Gel, respectively. For $T \leq 45{ }^{\circ} \mathrm{C}, G^{\prime}$ reaches a plateau with the values of 2.9 and $11.1 \mathrm{kPa}$ for $10 \%$ - and $20 \%$-Gels, respectively.

As discussed above, both PS- and PI-blocks' good solubility at high temperatures leads 
to a viscous solution. At low temperatures, the polymer network is formed because of physically associated PS-blocks connected by PI-blocks. ${ }^{1,2,6-8}$ A plateau in $G^{\prime}$ for both gels indicates the completion of network formation, and the microstructure is not expected to change significantly with the change of temperature in that region. A higher number of load-bearing chains in $20 \%$-Gel as a result of higher polymer concentration leads to a higher $G^{\prime}$ value than that for $10 \%$-Gel. Also, the higher number of chains facilitates the network formation at relatively higher temperature, therefore the $20 \%$-Gel has higher $T_{g e l}$.

$T_{\text {gel }}$ determined by shear-rheology generally varies with the applied frequency $(\omega)$, therefore, Winter-Chambon criterion is commonly implemented to identify the true gelation temperature. ${ }^{43-45}$ We have shown earlier that this gel does not follow Winter-Chambon criterion, similar to a few other physically assembled gels. ${ }^{8,46,47}$ It has been shown that for 10\%-Gel, the $G^{\prime}$ and $G^{\prime \prime}$ crossover shifts by $\approx 20{ }^{\circ} \mathrm{C}$ as $\omega$ increases from 0.1 to $100 \mathrm{rad} / \mathrm{s}^{8}$ Therefore, the $T_{g e l}$ indicated here can be considered as the apparent gelation temperature, which is expected to change with applied $\omega$. Note that the $T_{g e l} \mathrm{~S}$ are sufficiently higher than the room temperature, therefore, the microstructure formation at room temperature is expected to be complete.

For capturing the change in gel microstructure using RheoSAXS, the samples were heated sufficiently above their $T_{\text {gel }}$ and allowed to cool to $27^{\circ} \mathrm{C}$ by natural convection $(\approx 20 \mathrm{~min})$. Particularly, the $10 \%$-Gel sample was heated to $80{ }^{\circ} \mathrm{C}$ and the $20 \%$-Gel to $120{ }^{\circ} \mathrm{C}$, and was thermally equilibrated for $\approx 3 \mathrm{~min}$ at those temperatures. During cooling, the scattering data were collected at every $5{ }^{\circ} \mathrm{C}$ of temperature drop with an exposure time of $0.03 \mathrm{~s}$. As the sample was cooling continuously, there was a possibility of a small temperature gradient within the sample during the data collection. Figure 2B and $\mathrm{C}$ represent the circular averaged intensity $I(q)$ as a function of scattering vector $(q)$ for the $10 \%$ - and 20\%-Gels at different temperature. The 2D-scattering patterns presented in Figure 2D display a circular pattern indicating the isotropic gel microstructure as now was applied (i.e., static condition). Note that the elongated circle near the beam center captures the low- $q$ region $\left(<0.0002 \AA^{-1}\right)$ and 
(A)

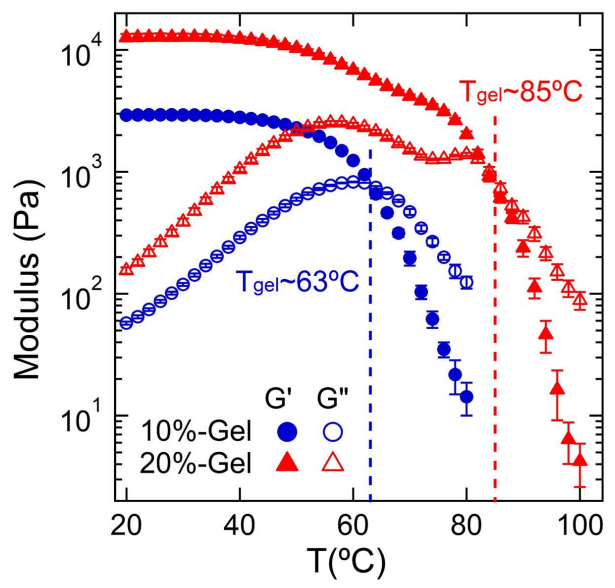

(C)

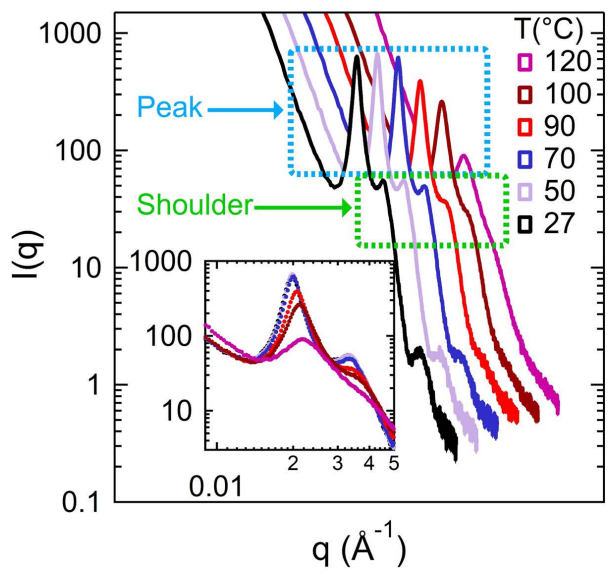

(B)

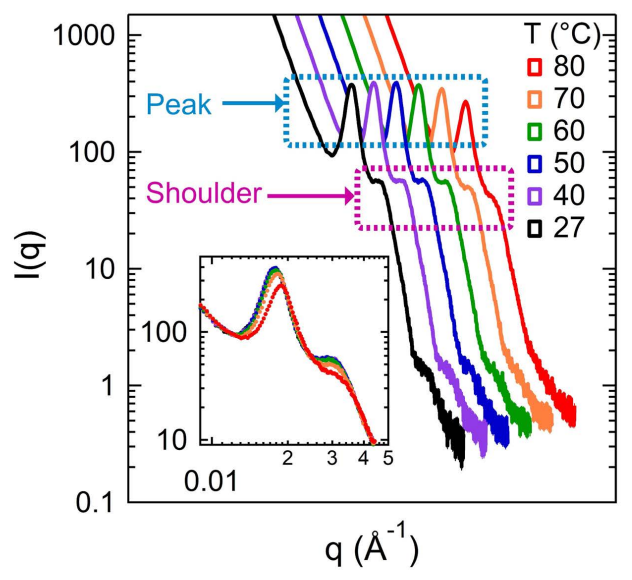

(D1)

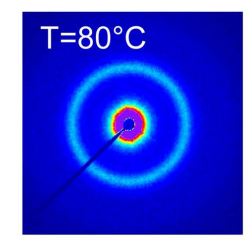

(D2)

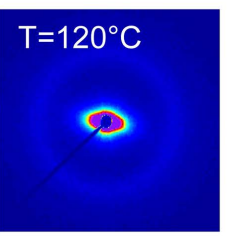

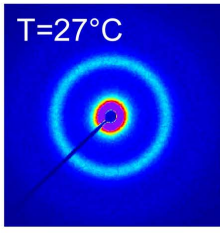

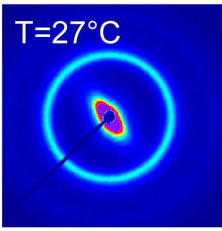

Figure 2: Temperature dependent gelation process. (A) Storage modulus $\left(G^{\prime}\right)$ and loss modulus $\left(G^{\prime \prime}\right)$ as a function of temperature $(T)$ for $10 \%$ - and 20\%-Gels captured using shear-rheology experiments with an oscillatory amplitude $\left(\gamma_{a m p}\right)$ of 0.01 and frequency $(\omega)$ of $1 \mathrm{rad} / \mathrm{s}$. A cooling rate of $2{ }^{\circ} \mathrm{C} / \mathrm{min}$ with a soak time of $300 \mathrm{~s}$ was applied. Intensity $(I(q))$ as a function of scattering angle $(q)$ for $(\mathrm{B}) 10 \%$-Gel at $27,40,50,60,70$, and $80{ }^{\circ} \mathrm{C}$, and for (C) 20\%-Gel at 27, 50, 70, 90, 100, and $120^{\circ} \mathrm{C}$. The curves are shifted along the $q$-axis for clarity. The insets in B and $\mathrm{C}$ represent the $I(q)$ vs. $q$ without shifting. The 2D-scattering patterns above $T_{\text {gel }}$ and at room temperature are shown for (D1) $10 \%$-Gel and (D2) 20\%-Gel.

is mostly an experimental artifact that has not been considered for the analysis.

For both gels, a decrease in $T$ causes the evolution of the microstructure, which is manifested by increased intensity and narrowing of the peak at $q \approx 0.02 \AA^{-1}$. Development of a weak shoulder at $q \approx 0.03 \AA^{-1}$ is also observed. This shoulder corresponds to a low-intensity 
circle in the 2D-scattering profiles (Figure 2D1-D2). The peak at $q \approx 0.02 \AA^{-1}$ is related to the structure factor (Bragg peak) capturing inter-aggregate scattering, and the shoulder at $q \approx 0.03 \AA^{-1}$ corresponds to the form factor of spherical PS-aggregates. ${ }^{6,7}$ At $T<45^{\circ} \mathrm{C}$, the scattering profiles overlap and those do not change significantly with a further reduction in $T$ (Figure 2B-C insets) indicating a developed microstructure, which is also evident from the plateau in $G^{\prime}$ (Figure $2 \mathrm{~A}$ ). The peaks beyond $q \approx 0.05 \AA^{-1}$ are secondary reflections of the form factor. ${ }^{48}$ Interestingly, the Bragg peak at $q \approx 0.02 \AA^{-1}$ and a weak shoulder at $q \approx 0.03 \AA^{-1}$ are also observed at $T \geq T_{\text {gel }}$ for both gels, although at a lower intensity than that observed below $T_{\text {gel }}$ (see Figure 2B). The initiation of the aggregate formation above the $T_{\text {gel }}$ supports the argument that the shear-rheology experiments capture an apparent gelation temperature. Also, gelation does not occur at a single temperature but takes place over a temperature range. A similar evolution of the structure and form factors with temperature had also been captured for acrylic gels. ${ }^{4,22}$ For the PS-PI-PS gels, $I(q) \propto q^{-3 \text { to }-3.2}$ over $0.0002<q<0.01 \AA^{-1}$ at all temperatures signifies a rough surface fractal similar to that observed for acrylic gels. ${ }^{22}$

A polydispersed core hard-sphere model with Percus-Yevick closure has been used to fit the scattering data for the $q$-range of $0.01 \leq q \leq 0.050 \AA^{-1} .4,6,7,22$ Mathematically, this model is expressed as, $I(q) \propto P(q) S_{1}(q)$, where $P(q)$ represents the spherical form factor and $S_{1}(q)$ the structure factor. As shown schematically in Figure S1, the PS aggregates are represented by cores with mean radius $r_{c}$ and polydispersity $\sigma / r_{c}$, where $\sigma$ is the standard deviation of $r_{c} \cdot{ }^{4,6-8,22}$ These aggregates are surrounded by a fictitious hard sphere with an average thickness $s_{1}$ and a volume fraction $\psi_{1}$. Considering that every hard-sphere is contained in a fictitious cube with a dimension $D$ (see Figure $\mathrm{S} 1$ ), the inter-aggregate distance can be estimated as, $D_{1}=\left(4 \pi\left(r_{c}+s_{1}\right)^{3} /\left(3 \psi_{1}\right)\right)^{1 / 3}$. $^{4}$ After subtracting the background, the features for $q>0.050 \AA^{-1}$ and $q<0.01 \AA^{-1}$ are not very discernible and have not been considered here for further analysis.

Figure $3 \mathrm{~A}$ compares the scattering profiles for the $10 \%$ - and $20 \%$-Gels at $27^{\circ} \mathrm{C}$. The solid 
(A)

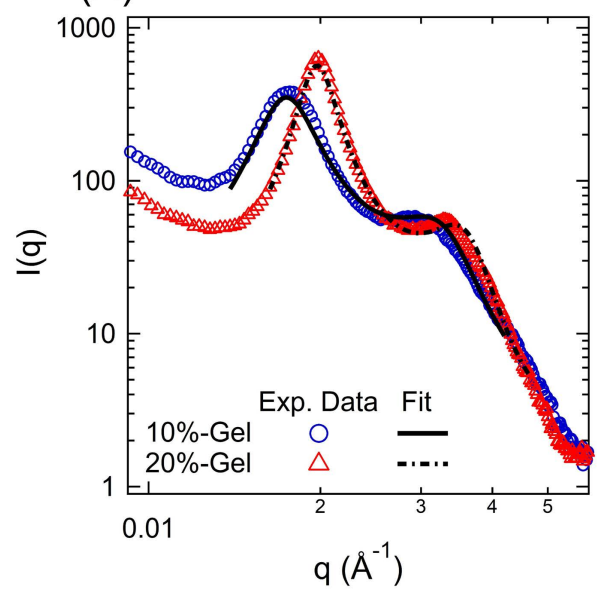

(B1)

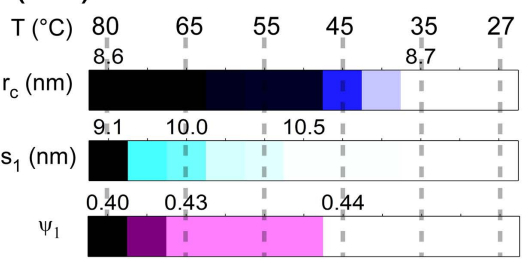

(B2)

$\mathrm{T}\left({ }^{\circ} \mathrm{C}\right) \begin{array}{lllllllll}120 & 95 & 85 & 75 & 65 & 55 & 45 & 35 & 27\end{array}$

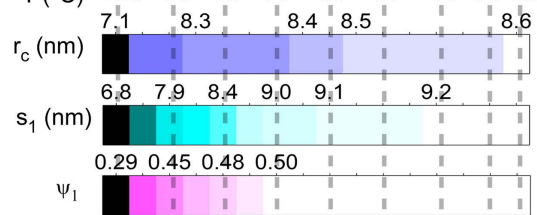

(C)
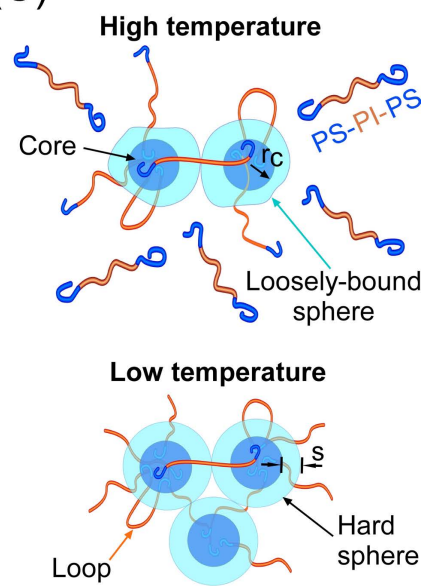

Figure 3: Evolution of gel microstructure as a function of temperature. (A) Comparison of $I(q))$ vs. $q$ for $10 \%$ - and $20 \%$-Gels at $T=27{ }^{\circ} \mathrm{C}$ (markers), and fitting with a polydispersed core hard-sphere model (lines). (B) Fitted parameters, viz. the core radius $\left(r_{c}\right)$, hardsphere thickness $\left(s_{1}\right)$, and hard-sphere volume fraction $\left(\psi_{1}\right)$ are presented as a function of temperature for the (B1) 10\%-Gel and (B2) 20\%-Gel. (C) Schematic representing the assembly of PS- and PI-blocks leading to the formation of network structure with the change of temperature.

lines represent the model fit, which capture the data reasonably well. Note that the structure factor peak for $10 \%$-Gel is at slightly lower $q$ than that for $20 \%$-Gel, indicating a higher interaggregate distance. The fitted parameters at different temperatures for both gels are shown in Figure 3B1-B2. These values are typical of the physically assembled gels in midblockselective solvents. ${ }^{1,2,4,6-8,22}$ For both gels, $r_{c}, s_{1}$, and $\psi_{1}$ gradually increase with decreasing $T$. For $10 \%$-Gel, $r_{c}$ remains almost constant $(\approx 8.6 \mathrm{~nm}), s_{1}$ increases from 9.1 to $10.5 \mathrm{~nm}$, and $\psi_{1}$ increases from 0.4 to 0.44 as $T$ decreases from 80 to $27^{\circ} \mathrm{C}$. Similarly, for $20 \%$ gel, $r_{c}$ increases from $7.1 \mathrm{~nm}$ to $8.6 \mathrm{~nm}, s_{1}$ increases from 6.8 to $9.2 \mathrm{~nm}$, and $\psi_{1}$ increases from 0.29 to 0.50 while decreasing $T$ from 120 to $27^{\circ} \mathrm{C}$. For both gels, the microstructure evolves and moduli increase with decreasing temperature, but the microstructural change is not significant once the $G^{\prime}$ of these gels attains a plateau (compare Figure 2A and Figure 3B1-B2).

With decreasing $T$, the PS-aggregates expel the solvent, ${ }^{3,4}$ and this process can lead to the decrease of the aggregates radii. However, our data suggest that $r_{c}$ increases slightly, especially in 20\%-Gel. It can be hypothesized that at higher $T$, the PS-blocks loosely as- 
sociate to form smaller aggregates. Also, dangling PS-chains that are not connected to any aggregate also exist (Figure 3C). This is also evident from not so well-developed form factor shoulders (weaker intensity) in Figure 2B-C. With the decrease in $T$, those non-connected PS-blocks merge in the existing bigger aggregates leading to an increase of $r_{c}$. The space between two aggregates also increases slightly to accommodate the PI chains, leading to an increase in $s_{1}$.

In addition to the density of midblocks acting as bridges, the midblock stretch also contributes to the small strain modulus of the gels. ${ }^{4,7}$ The length of PI-block in the gels can be theoretically estimated by considering the mineral oil as a $\theta$-solvent at $27^{\circ} \mathrm{C} \cdot{ }^{6,7}$ The end-to-end distance of a PI-block in $\theta$-solvent can be calculated as, $R_{e, P I}=b \sqrt{N} \approx 21.6 \mathrm{~nm}$, where $b(\approx 0.84 \mathrm{~nm})$ and $N(\approx 662)$ are the length and number of Kuhn segments per PIblock, respectively. ${ }^{6,7,49}$ We estimate the stretch ratio, $\left(D_{1}-2 r_{c}\right) / R_{e, P I}$, for the $10 \%$ - and $20 \%$-Gel as $\approx 1.08$ and 0.88 , respectively. This suggests the PI-blocks are slightly stretched in 10\%-Gel. In reality, mineral oil is a good solvent to the PI-blocks, and that can lead to a higher value of $R_{e, P I},{ }^{1}$ therefore, the PI-blocks in 10\%-Gel may not be stretched as predicted for $\theta$-solvent. Similarly, the PI-blocks in $20 \%$-Gel are expected to be more compressed in mineral oil. The compressed chains signify that the gels can achieve a higher stretch ratio before failure. ${ }^{6,7}$

\section{Microstructural Change with Oscillatory Shear}

The effect of shear deformation on the gel microstructure was explored using oscillatory shear experiments. Figure $4 \mathrm{~A}$ displays $G^{\prime}$ and $G^{\prime \prime}$ for $10 \%$ - and $20 \%$-Gels obtained from amplitude sweep experiments at $27^{\circ} \mathrm{C}$. The strain-amplitude $\left(\gamma_{a m p}\right)$ was varied over the range of $10^{-4}$ to 1 using $\omega$ of $1 \mathrm{rad} / \mathrm{s}$. Over this range, the gel samples did not fail, and no noticeable change in $G^{\prime}$ and $G^{\prime \prime}$ was observed. The stress-controlled rheometer used in this study could not correct the phase-angle accurately beyond $\gamma_{a m p}>1$, thus, those data have not been considered here. 
(A)

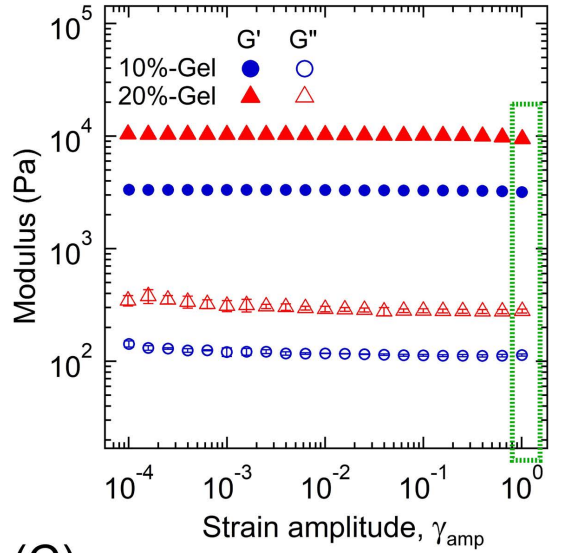

(C)
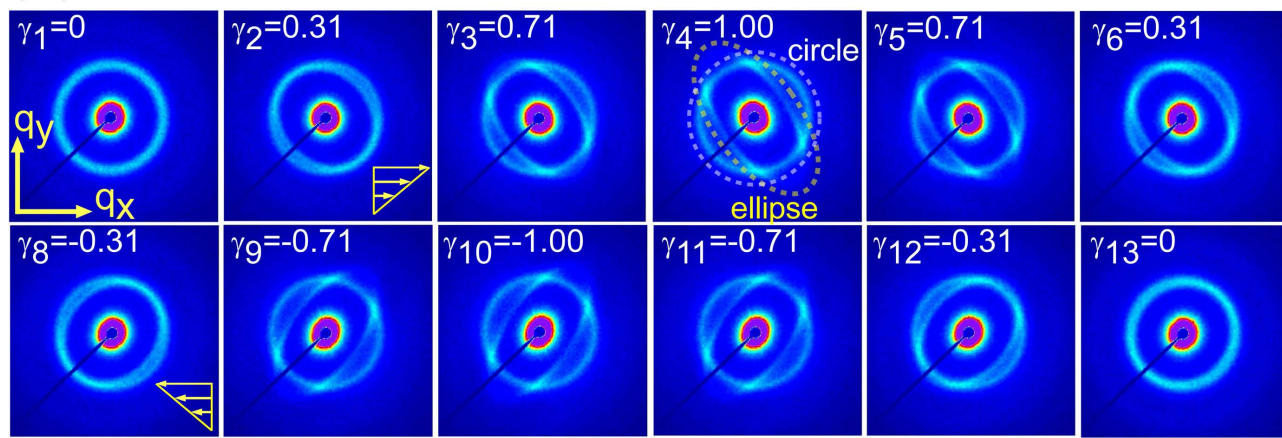

(D)
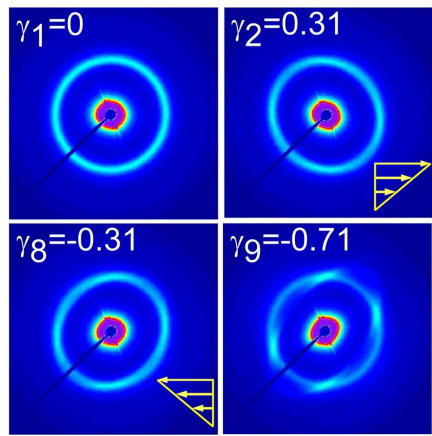

(B)

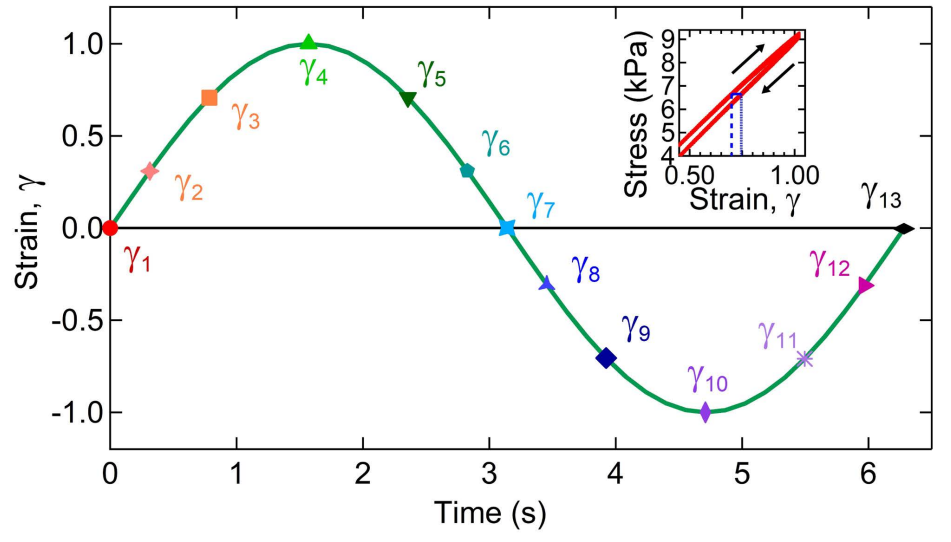

Figure 4: Shear-rheology and RheoSAXS results for oscillatory shear experiments at $27^{\circ} \mathrm{C}$. (A) Storage modulus $\left(G^{\prime}\right)$ and loss modulus $\left(G^{\prime \prime}\right)$ as a function of strain amplitude $\left(\gamma_{a m p}\right)$ for 10\%- and 20\%-Gels using a frequency $(\omega)$ of $1 \mathrm{rad} / \mathrm{s}$. The results for $\gamma_{a m p}=1$ are highlighted. The error bars, smaller than the markers in some cases, represent one standard deviation. (B) Schematic of one oscillatory strain cycle as a function of time for $\gamma_{a m p}=1$ at $\omega=1 \mathrm{rad} / \mathrm{s}$. The inset displays the stress vs. strain plot for $20 \%$-Gel displaying a small hysteresis of the gel. The markers on the strain cycle correspond to the 2D-scattering patterns presented in (C) for 10\%-Gel, and (D) for 20\%-Gel. In C and D, $q_{x}$ represents $v$ or flow direction and $q_{y}$ represents $\nabla v$ or velocity gradient direction. 
In RheoSAXS experiments, the 10\%-Gel was subjected to oscillatory strain-amplitude of $\gamma_{a m p}=0.1$ at $27^{\circ} \mathrm{C}$, and $\gamma_{a m p}=1$ at $27^{\circ} \mathrm{C}$ and $40{ }^{\circ} \mathrm{C}$, respectively. Similarly, the $20 \%$-Gel was subjected to $\gamma_{a m p}=0.1$ and 1 at $27^{\circ} \mathrm{C}$. All these experiments were conducted using $\omega=1 \mathrm{rad} / \mathrm{s}$. For the experiments at $27^{\circ} \mathrm{C}$, three cycles with $\gamma_{a m p}=0.1$ were applied, followed by three cycles with $\gamma_{a m p}=1$. The scattering data were collected during three oscillatory cycles with an exposure time of 0.03 and $0.05 \mathrm{~s}$ for the $10 \%$ - and the $20 \%$-Gel, respectively. With such a short exposure time, we estimate a maximum $\approx 5 \%$ change in intracycle strain value while collecting the data. The time interval between two data points was $0.157 \mathrm{~s}$, resulting in $\approx 40$ data points over a strain cycle. Figure $4 \mathrm{~B}$ represents a strain cycle for $\gamma_{a m p}=1$ in which thirteen representative intracycle strain values $\left(\gamma_{1}-\gamma_{13}\right)$ are selected. The 2Dscattering patterns corresponding to these strain values for 10\%- and 20\%-Gels are displayed in Figure $4 \mathrm{C}$ and D. Two movies capturing the 2D-scattering pattern as a function of strain over three strain cycles for 10\%- and 20\%-Gels are shown in SI (10\%Gel_27C_Strain1.avi, 20\%Gel_27C_Strain1.avi). The inset in Figure 4B inset represents the stress vs. strain data for $20 \%$-Gel in which a small hysteresis of the gel can be noted.

A particular strain-cycle can be divided into four quarters, for $\gamma=0$ to $+1,+1$ to 0,0 to -1 , and -1 to 0 . For both gels, at $\gamma_{1}(=0)$, the scattering pattern is circular, indicating isotropic gel microstructure at the static condition (Figure 4C and D). ${ }^{4,6,7,22}$ For 10\%-Gel, in the first quarter, as the strain increases, the circular pattern transforms to an elliptical pattern having clear major and minor axes (for example, $\gamma_{2}=0.31$ ). The elliptical pattern splits into two parts, elliptical and circular patterns, with increasing strain, as presented for $\gamma_{3}=0.71$. The circular pattern has the same radius as that obtained for $\gamma_{1}=0$. With increasing strain to $\gamma_{4}=1$, the split becomes more prominent. Interestingly, the $q$-value corresponding to the intersection of circular and elliptical profiles displays higher intensity relative to the other $q$-values. This intensity is similar to that observed for all $q$-values at $\gamma_{1}$.

In the second quarter, as the strain values decrease to 0 , the split pattern transforms to a purely elliptical pattern and then to a circular pattern at $\gamma_{7}=0$. In the 3 rd quarter, the 
(A1)

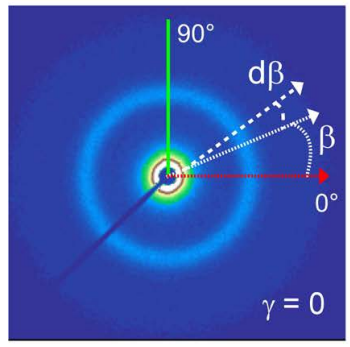

(B)

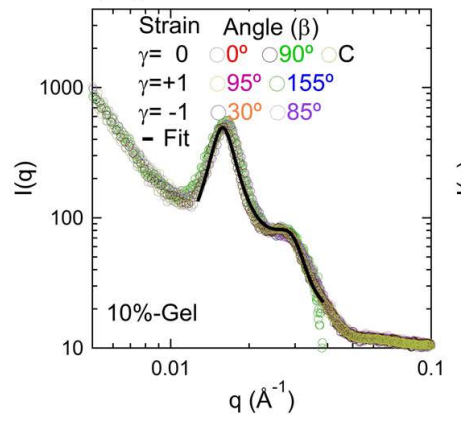

(A2)

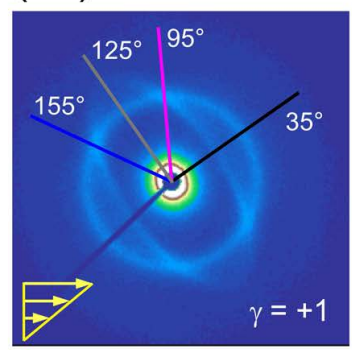

(C)

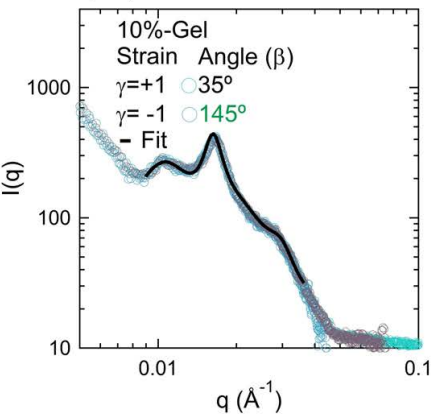

(A3)

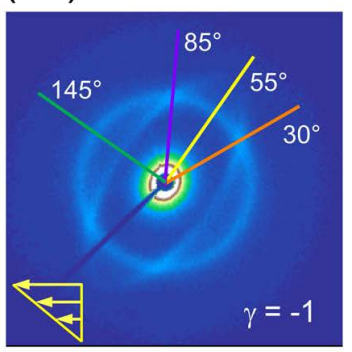

(D)

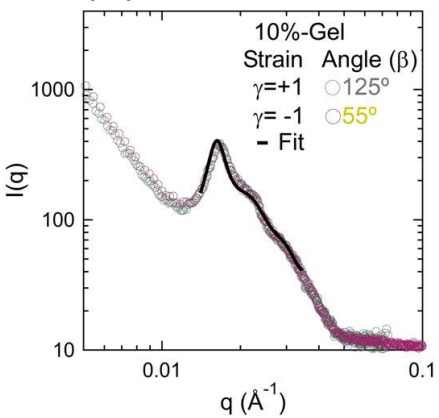

Figure 5: Scattering patterns and $I(q)-q$ plots for $10 \%$-Gel at $27^{\circ} \mathrm{C}$ for the strain amplitude of 1 and for the static condition. The azimuthal angle $\beta$ is defined in (A1). The 2D-scattering patterns are shown for (A1) $\gamma=0$, (A2) $\gamma=+1$ (clockwise), and (A3) $\gamma=-1$ (anticlockwise). 1D-scattering patterns and model fit are shown for (B) circular pattern and intersection between circle and ellipse, (C) minor-axis of the ellipse, and (D) major-axis of the ellipse.

scattering pattern resembles that of the 1st quarter, however, the angle of orientation of the elliptical pattern changes because of the change in applied strain direction in an oscillatory cycle. Further, the data for 2 nd and 4 th quarters are similar. Note that scattering patterns for $\gamma=0.31$ in the 1 st and 2 nd quarters, i.e., for $\gamma_{2}$ and $\gamma_{6}$, are slightly different. We relate that to the slight hysteresis in the strain cycle (Figure 4B inset). Note that for the same stress values $(\approx 6.7 \mathrm{kPa})$ there are two different $\gamma$ values $(\approx 0.71$ and $\approx 0.75)$, which may have affected the symmetry of the 2D-scattering pattern with respect to $\gamma$. For 20\%-Gel, the strain-dependent scattering profiles are very similar to those of $10 \%$-Gel, except that the split elliptical pattern is more prominent in 10\%-Gel (Figure 4D). 2D-scattering profiles are also represented for both gels for $\gamma_{a m p}=0.1$ in Figure S2. For this low strain amplitude, no split in 2D-pattern was observed.

Figure 5A1-A3 displays the 2D-scattering patterns for 10\%-Gel at the intracycle strain of 
$\gamma=0,+1$, and -1 . The corresponding $I(q)$ vs. $q$ for different $\beta$ values have been plotted by averaging $I(q)$ over $\beta \pm 5^{\circ}$ sector (Figure 5B-D). Here, $\beta$ corresponds to the angle measured from the $q_{x}$-axis or flow direction (Figure 5A). As shown in Figure 5B, the scattering profiles at $\beta \approx 95^{\circ}$ and $155^{\circ}$ for $\gamma=+1$, at $\beta \approx 30^{\circ}$ and $85^{\circ}$ for $\gamma=-1$, and at $\beta \approx 0^{\circ}$ and $90^{\circ}$ for $\gamma=0$ overlap. For $\gamma= \pm 1$, these $\beta$ values represent the intersection between elliptical and circular patterns. Along the minor-axis of the ellipitical pattern, i.e., the scattering profiles at $\beta \approx 35^{\circ}$ for $\gamma=+1$ (Figure 5A2) and at $\beta \approx 145^{\circ}$ for $\gamma=-1$ (Figure 5A3) overlap, as shown in Figure 5C. Similarly, as presented in Figure 5D, the scattering profiles along the major-axis of the elliptical pattern, i.e., at $\beta \approx 125^{\circ}$ for $\gamma=+1$ (Figure $5 \mathrm{~A} 2$ ), and at $\beta \approx 55^{\circ}$ for $\gamma=-1$ (Figure 5A3) overlap.

The $I(q)-q$ plots along the minor-axis of the ellipse for $\gamma= \pm 1$ display an additional peak at $q \approx 0.01 \AA^{-1}$ (Figure $5 \mathrm{C}$ ) compared to that for $\gamma=0$. Also, a slight decrease in the intensity of the peak at $q \approx 0.017 \AA^{-1}$ can be noticed. A similar comparison between the $I(q)-q$ plots along the major-axis of the ellipse for $\gamma= \pm 1$, and that for $\gamma=0$ also indicates an additional peak at $q \approx 0.023 \AA^{-1}$ (Figure 5D). However, this peak is in the vicinity of the form factor shoulder and is not clearly discernible. The decrease in the intensity of the structure factor peak is associated with the emergence of the additional peak signifying the change in inter-aggregate distance for some aggregates. In other words, the additional peak is also a structure factor peak with different inter-aggregate distance. As shown in Figure 5C-D, both elliptical and circular patterns are observed at $\gamma= \pm 1$. Further, the position and the intensity of the form factor shoulder remain similar for all $\gamma$ values. The unchanged form factor peak signifies that the aggregates' shape and size have not changed in the $\gamma$-range considered here. Similar behavior has also been observed for the $20 \%$-Gel presented in Figure 6A-D. As shown in Figure 6B-D, at $\gamma==+1$ and -1 , additional peaks appear at $q \approx 0.013 \AA^{-1}$ and $q \approx 0.025 \AA^{-1}$, respectively. However, these additional peaks are not as prominent as for the case of $10 \%$-Gel.

The overlapping data for $\gamma= \pm 1$ shown above indicate that the microstructure change 
(A1)

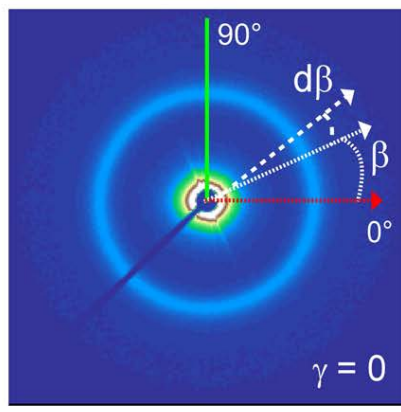

(B)

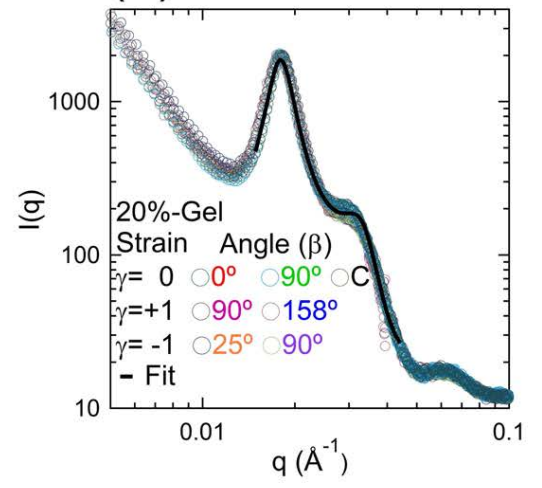

(A2)

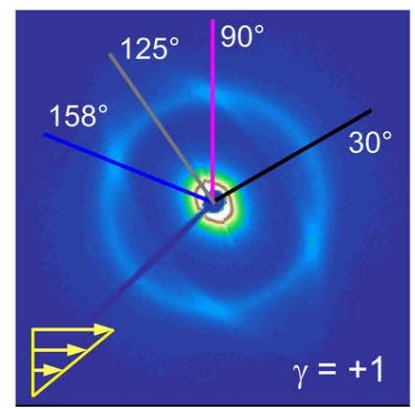

(C)

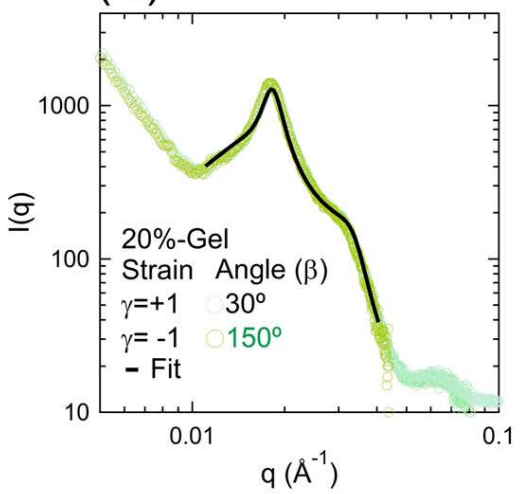

(A3)

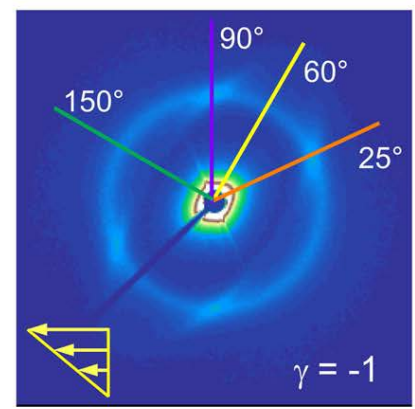

(D)

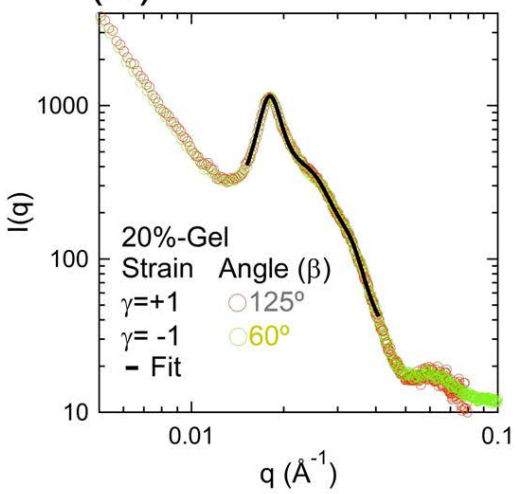

Figure 6: Scattering patterns and $I(q)-q$ plots for $20 \%$-Gel at $27{ }^{\circ} \mathrm{C}$ for the strain amplitude of 1 and for the static condition. 2D-scattering patterns are shown for (A1) $\gamma=0,(\mathrm{~A} 2) \gamma=+1$ (clockwise), and (A3) $\gamma=-1$ (anticlockwise). 1D-scattering patterns and model fit are shown for (B) circular pattern and intersection between circle and ellipse, (C) minor-axis of the ellipse, and (D) major-axis of the ellipse.

during a strain cycle is independent of $\gamma$ direction and solely depends on its magnitude $(|\gamma|)$. Fitting a model to the extra peak of the $I(q)-q$ data along the minor and major axes of the elliptical pattern can provide additional insights regarding the change in gel microstructure with $\gamma$. As the aggregates remain the same, the scattering data in Figure $5 \mathrm{~B}$ and $6 \mathrm{~B}$ can be fitted with the same polydispersed core hard-sphere model described above for the static samples. A modified form of the polydispersed core hard-sphere model, mathematically expressed as $I(q) \sim P(q)\left(S_{1}(q)+S_{2}(q)\right)$ has been used. Here, $S_{1}(q)$ represents the structure factor corresponding to the circular pattern (or intersection). $S_{2}(q)$ represents the secondary structure factor corresponding to the elliptical pattern capturing the new peak. Note that the form factor $(P(q))$ is the same for $S_{1}(q)$ and $S_{2}(q)$, capturing that the features of the PSaggregates do not change upon deformation. Note that this model results in two hard-sphere 
volume fractions, $\psi_{1}$ and $\psi_{2}$, because of change in the inter-aggregate distance.

The model fittings are shown in Figure 5B-D and 6B-D for 10\%- and 20\%-Gels, respectively, and the fitted parameters are presented in Table 1 . The $r_{c}$ values for 10\%- and 20\%-Gels in Figure 5B and 6B are slightly higher than those observed in temperature sweep experiments presented in Figure $3 \mathrm{~B}$ at $27^{\circ} \mathrm{C}$. This is possibly due to the different cooling cycled allowed in both experiments. Here, the samples were cooled by blowing air for $\approx 30$ min while the data related to Figure 3 was collected during natural cooling of the gels ( $\approx 20 \mathrm{~min})$. Note that the difference in the $r_{c}$ values is not significant, as the change is within the limit of polydispersity $\left(\sigma / r_{c} \approx 0.2\right)$.

Table 1: Parameters obtained from fitting scattering data to polydispersed core hard-sphere model with Percus-Yevick closure for three different sectors at $\gamma=0,+1$ and -1 .

\begin{tabular}{cccccccc}
\hline Sample & Sector & $r_{c}(\mathrm{~nm})$ & $\sigma / r_{0}$ & $s_{1}(\mathrm{~nm})$ & $\psi_{1}$ & $s_{2}(\mathrm{~nm})$ & $\psi_{2}$ \\
\hline \multirow{2}{*}{$10 \%$-Gel } & intersection & 10.2 & 0.01 & 11.0 & 0.43 & - & - \\
& minor-axis & 10.2 & 0.17 & 11.0 & 0.48 & 18.8 & 0.27 \\
& major-axis & 10.2 & 0.30 & 11.0 & 0.48 & 4.0 & 0.33 \\
\hline \multirow{2}{*}{$20 \%$-Gel } & intersection & 9.3 & 0.19 & 9.6 & 0.46 & - & - \\
& minor-axis & 9.3 & 0.10 & 9.6 & 0.48 & 9.8 & 0.19 \\
& major-axis & 9.3 & 0.28 & 9.6 & 0.47 & 2.9 & 0.30 \\
\hline
\end{tabular}

For the $10 \%$-Gel, the inter-aggregate distance $\left(D_{1}\right)$ at the static condition $(\gamma=0)$ is estimated as $\approx 45.3 \mathrm{~nm}$. For $|\gamma|=1$, two additional inter-aggregate distances $\left(D_{2}\right)$ can be estimated corresponding to the major- and minor-axes. $D_{2}$ along the minor- and major-axes is expected to capture the inter-aggregate distance for the displaced aggregates because of applied strain (see Figure S3). Similar to $D_{1}, D_{2}$ can be estimated as $\left(4 \pi\left(r_{c}+s_{2}\right)^{3} /\left(3 \psi_{2}\right)\right)^{1 / 3}$ using the fitted parameters $s_{2}$ and $\psi_{2}$. We obtain $D_{1} \approx 43.6 \mathrm{~nm}$ from the circular profiles which is observed in addition to the elliptical profiles for $|\gamma|=1$, resembling the isotropic case of $\gamma=0$. The $D_{2}$ values corresponding to the major- and minor-axis are $\approx 33.1 \mathrm{~nm}$ and $72.3 \mathrm{~nm}$, respectively. Similarly, for $20 \%-\mathrm{Gel}, D_{1}$ is $\approx 39.2 \mathrm{~nm}$, and $D_{2}$ values are $\approx 53.6 \mathrm{~nm}$ and $\approx 29.4 \mathrm{~nm}$ for the minor- and major-axes, respectively. The lower and higher $D_{2}$ values represent the decrease and increase in the inter-aggregate distance, respectively. 
With the applied $\gamma$, the inter-aggregate distance (or hard-sphere thickness) increases in the stretch direction. As a result, the inter-aggregate distance in the orthogonal direction decreases. The corresponding stretch or compression ratios can be estimated as $D_{2} / D_{1}$. We estimate the stretch and compression ratios are $\approx 1.66$ and $\approx 0.76$ for $10 \%$-Gel, and $\approx 1.38$ and $\approx 0.75$ for $20 \%$-Gel, respectively. The scattering data implies that the compression ratios are similar for these gels, whereas the stretch ratios are slightly different. These ratios can be related to the applied macroscopic deformation. For affine deformation of polymer chains, $\gamma$ can be theoretically related to the uniaxial stretch/compression ratio $(\lambda)$ as $\gamma=\lambda-\lambda^{-1} .50$ For $\gamma=1$, this relationship provides $\lambda=1.62$ and -0.62 , where the positive and negative signs indicate stretching and compression, respectively. The theoretical stretch ratio is similar to that obtained from the scattering data for $10 \%$-Gel, but it is slightly higher than that for $20 \%$ Gel. The compression ratios for the gels are slightly higher than that estimated theoretically. For the case of $20 \%$-Gel, a deviation in the stretch ratio than that obtained theoretically can be attributed to the relatively higher inhomogeneity in the microstructure, likely aided by the slightly entangled PI-blocks. ${ }^{6,7}$ In addition, thermal quenching protocol used here can also cause higher inhomogeneity in the gel microstructure. These local variations in the microstructure can be viewed as defects, which can cause strain localization, as reported for the acrylic gels. ${ }^{51,52}$

In summary, the oscillatory shear-rheology results indicate that the PI chains are stretched in the flow direction and are compressed in the orthogonal direction. The stretch and compression ratios are similar to those predicted theoretically. An oriented microstructure results in an elliptical 2D-scattering pattern. However, the presence of both elliptical and circular patterns at larger intracycle strain indicates that some of the aggregates have retracted to their unperturbed condition. We hypothesize the strain-localization in these samples, ${ }^{51,52}$ where the local strain values can be higher than the macroscopic strain value. Such higher local strain values facilitate the PS-chain pullout from the aggregates. As a result, those aggregates retract to the unstretched condition. It can also be argued that such pullout 
weakens the non-linear behavior like strain-stiffening responses, which are prominently observed in the acrylic gels. ${ }^{24,53}$ For acrylic gels, these strain-stiffening responses were related to the maximum extensibility of midblocks before being pulled out from the aggregates. Note that we have not observed any clear sign of non-linearity from the rheological responses of the gels investigated here, i.e., the $e_{3}$ and $\nu_{3}$ values are almost zero. ${ }^{53-57}$ It may be possible that because of local inhomogeneity individual PI-block may have reached the maximum stretchability, but collectively not displaying strain-stiffening response. Also, weaker association of PS blocks in comparison to that of PMMA block in the acrlyic gels cannot be ruled out. Further investigations will be necessary to identify the correct reasons.

\section{Stress-Relaxation Behavior}

The viscoelastic nature of these gels causes stress dissipation when subjected to load. This dissipation process can be quantified through stress-relaxation experiments. In these experiments, a step shear strain $\left(\gamma_{\text {step }}\right)$ was applied on the gels, and the samples were then allowed to relax. The time-dependent stress decay $(\sigma(t))$ was recorded during the relaxation process and the corresponding time-dependent shear modulus $(G(t))$ can be estimated as $\sigma(t) / \gamma_{\text {step }}$. The corresponding change in microstructure was investigated from RheoSAXS experiments. Figure 7A1 represents the stress-relaxation behavior of a $10 \%$-Gel at $40{ }^{\circ} \mathrm{C}$ over $600 \mathrm{~s}$ for $\gamma_{\text {step }}=0.3$. The relaxation behavior for $0.1 \leq t \leq 1800$ is shown in Figure S4. However, data only up to $600 \mathrm{~s}$ is presented here to compare with the RheoSAXS data. Similarly, for $20 \%$-Gel, $G(t)$ was obtained at 50 and $70{ }^{\circ} \mathrm{C}$ (Figure $7 \mathrm{~B} 1-\mathrm{C} 1$ ). Note that for $70{ }^{\circ} \mathrm{C}$, the relaxation process is quite fast, resulting in a steeper decrease of $G(t)$. The experiments were also conducted for both gels at $27{ }^{\circ} \mathrm{C}$ where a smooth decay of $G(t)$ is observed (see Figure S6). For each case, $G(t)$ reaches a plateau after the relaxation process, indicating an equilibrium modulus. At higher $T$ and lower polymer concentration, a relatively steeper decrease of $G(t)$ has been noticed.

RheoSAXS experiments were conducted at the same temperatures indicated above and 
(A1)

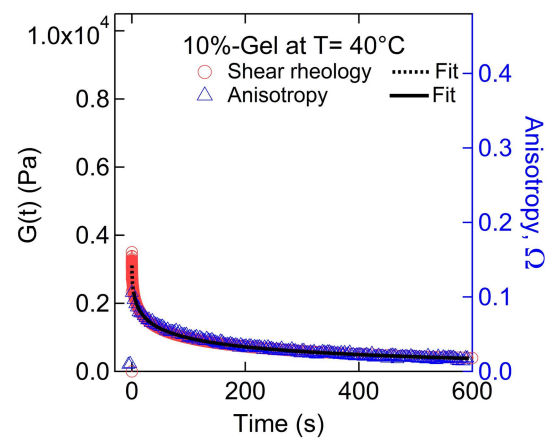

(A2)

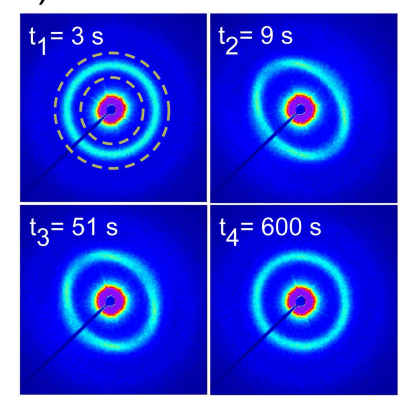

(A3)

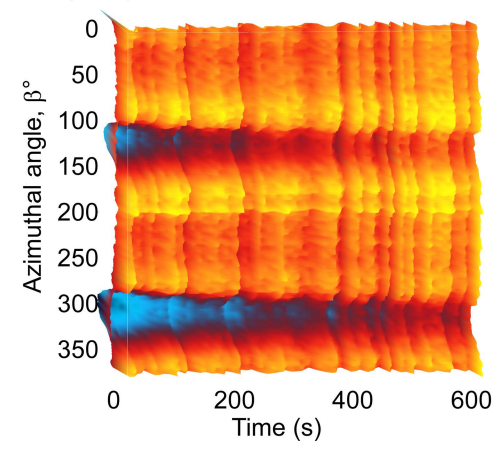

(B1)

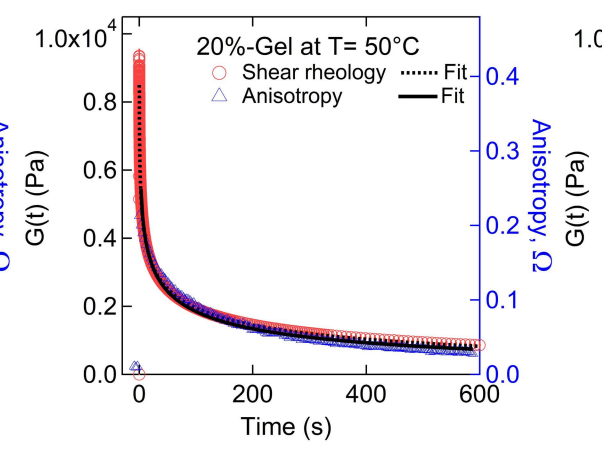

(B2)

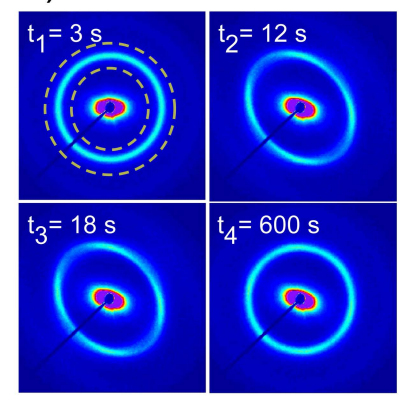

(B3)

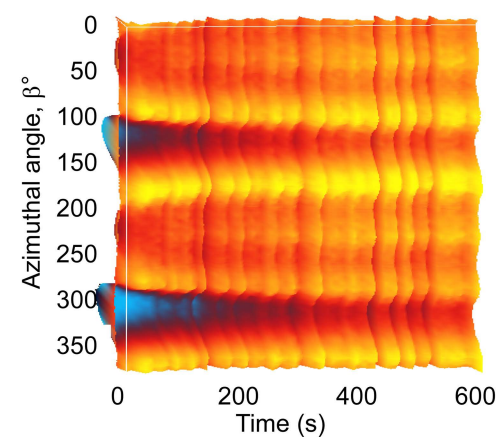

(C1)

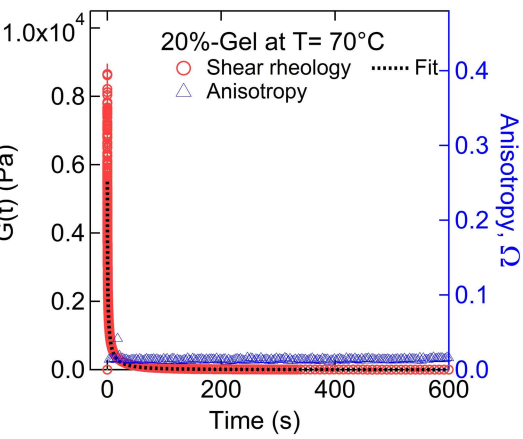

(C2)

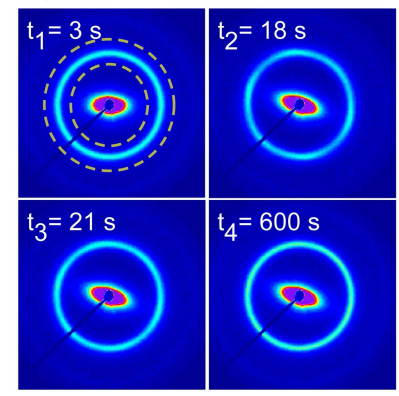

(C3)

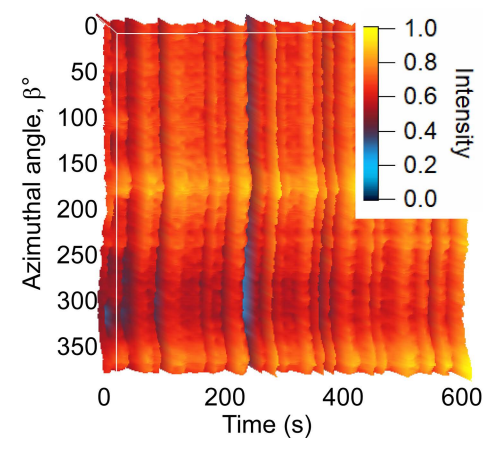

Figure 7: Stress-relaxation behavior obtained from shear-rheology and RheoSAXS experiments. Data for the applied step strain of 0.3 for the (A) $10 \%$-Gel at $40{ }^{\circ} \mathrm{C}$, and $20 \%$-Gel at (B) $50{ }^{\circ} \mathrm{C}$ and $(\mathrm{C}) 70^{\circ} \mathrm{C}$. (A1, B1, C1) Time-dependent modulus $(G(t)$ ), obtained from shear-rheology experiments, and anisotropy factor $(\Omega)$ estimated from RheoSAXS experiments. Lines represent stretched exponential model fitted to $G(t)$ and $\Omega$ data. (A2, B2, C2) 2D-scattering patterns before the application of strain $\left(t_{1}\right)$, after the application of strain $\left(t_{2}\right)$, after $t=\tau_{S R}$ determined from stress-relaxation data $\left(t_{3}\right)$, and at 600s $\left(t_{4}\right)$. The $q-$ range considered for the calculation of anisotropy factor is indicated by two dashed-circles in each $t_{1}$-image. For model fitting, the time-scale was shifted appropriately. (A3, B3, C3) Azimuthal plots as a function of time for different $\beta$ values are shown.

$\gamma_{s t e p}=0.3$ was applied. The scattering data were collected for $600 \mathrm{~s}$ with an X-ray exposure time of $0.03 \mathrm{~s}$ at an interval of $3 \mathrm{~s}$. 2D-scattering profiles at different time points during 
the relaxation process are displayed in Figure 7A2-C2. Here, $t_{1}$ displays the circular pattern at the static condition. At $t_{2}$, an elliptical pattern is observed representing a stretched or oriented structure as a result of applied $\gamma_{\text {step }}$. Figure S5 represents the $I(q)-q$ data before and after the application of strain for $20 \%$-Gel at $50{ }^{\circ} \mathrm{C}$. Here, we have compared the data along major- and minor-axes with that of the static condition. The shifting of structure factor peak to lower- $q$ along the elliptical pattern's minor axis represents the sample stretch. Similarly, the data along the major-axis signifies the compression of those in the orthogonal direction. The form-factor peak display a slight change in intensity, however, the $q$-position does not change appreciably. Similar to that presented in the previous section, fitting the scattering data with polydispersed core hard-sphere model estimates stretching and compression ratios along the minor- and major-axes as 1.15 and 0.95 , respectively. These values are comparable to the theoretical prediction of 1.16 and 0.86 , respectively. For the $20 \%$-Gel at $70{ }^{\circ} \mathrm{C}$ (Figure $7 \mathrm{C} 2-\mathrm{C} 3$ ), the relaxation process was very fast and the elliptical scattering pattern was captured for only one frame.

An azimuthal plot can be created by plotting average intensity over a specified $q$-range, i.e., $I(\beta)$, for $\beta$ values from to 0 to $359^{\circ}$ at any particular time point. As defined above, $\beta$ is measured from the flow/stretch direction (Figures $5 \mathrm{~A}$ and $6 \mathrm{~A}$ ). The average intensity values are estimated by taking an average over the $q$-ranges of $\approx 0.015-0.018 \AA^{-1}$ for $10 \%$-Gel at $40{ }^{\circ} \mathrm{C}$, and $\approx 0.017-0.021 \AA^{-1}$ for $20 \%$-Gel at 50 and $70{ }^{\circ} \mathrm{C}$. Since the form factor peak position and intensity almost remain the same during the relaxation process, the $q$-ranges are chosen to encompass the change in structure-factor peak position. Figure 7A3-C3 displays the timelapsed azimuthal plots over $600 \mathrm{~s}$. The azimuthal plot captures the peaks corresponding to the oriented elliptical pattern having uneven intensity distribution. These peaks start to fade with time, indicating a restoration of its isotropic microstructure. No significant change in azimuthal plots and 2D-scattering patterns are observed at $27^{\circ} \mathrm{C}$ (Figure S6) over $600 \mathrm{~s}$ indicating a slower microstructural relaxation process.

The degree of microstructure orientation can be quantified by estimating the anisotropic 
factor $(\Omega)$. The $\Omega$ values are displayed in the right axis of Figure 7A1-C1. A point in the 2D-scattering plot can be represented in the form of a unit vector, $\vec{u}=u_{x} i+u_{y} j$, where $u_{x}$ and $u_{y}$ represent the components of $I(\beta)$ parallel and perpendicular to the flow direction. The $\Omega$ can be mathematically expressed as: $:^{30,35,58,59}$

$$
\Omega=\left[\left(\left\langle u_{x}^{2}\right\rangle-\left\langle u_{y}^{2}\right\rangle\right)^{2}+4\left\langle u_{x} u_{y}\right\rangle^{2}\right]^{1 / 2}
$$

where $\langle\ldots\rangle$ represents $I(\beta)$ weighted average quantity as,

$$
\left\langle u_{x}^{2}\right\rangle=\frac{\int_{0}^{2 \pi} I(\beta) \cos ^{2} \beta d \beta}{\int_{0}^{2 \pi} I(\beta) d \beta},\left\langle u_{y}^{2}\right\rangle=\frac{\int_{0}^{2 \pi} I(\beta) \sin ^{2} \beta d \beta}{\int_{0}^{2 \pi} I(\beta) d \beta} \text {, and }\left\langle u_{x} u_{y}\right\rangle=\frac{\int_{0}^{2 \pi} I(\beta) \cos \beta \sin \beta d \beta}{\int_{0}^{2 \pi} I(\beta) d \beta}
$$

Before the application of strain, $\Omega$ is as low as $\approx 0.020$ for all the cases. The $\Omega$ increases with the application of step strain as the oriented elliptical pattern emerges. At higher $T$, the increase of $\Omega$ is relatively higher (see Figure S6A-B). For example, for the $10 \%$-Gel at $40{ }^{\circ} \mathrm{C}, \Omega$ jumps from $\approx 0.01$ to 0.1 . Such behavior is due to the higher mobility of chains at elevated $T$ facilitating the orientation development. As shown in Figure 7A1-C1, with time $\Omega$ decreases for all cases, a trend similar to $G(t)$. Due to the faster relaxation of $20 \%$-Gel at $70{ }^{\circ} \mathrm{C}$, the $\Omega$ value jumps from 0.041 to 0.010 rapidly.

Table 2: Parameters obtained by fitting the stretched exponential function to the stressrelaxation and anisotropy factor data for $10 \%$ - and $20 \%$-Gels at different temperatures. Here $T$ represents experimental temperature, $\alpha$ stretch parameter, $G_{0}$ zero-time shear modulus, $\tau_{S R}$ stress-relaxation time, $\Omega_{0}$ anisotropy at $t \approx 0$, i.e., after the application of strain, and $\tau_{\Omega}$ microstructure recovery time.

\begin{tabular}{ccccccc}
\hline & & & \multicolumn{2}{c}{ Shear-rheology } & \multicolumn{2}{c}{ RheoSAXS } \\
Sample & $T\left({ }^{\circ} \mathrm{C}\right)$ & $\alpha$ & $G_{0}(\mathrm{~Pa})$ & $\tau_{S R}(\mathrm{~s})$ & $\Omega_{0}$ & $\tau_{\Omega}(\mathrm{s})$ \\
\hline 10\%-Gel & 27 & $0.30 \pm 0.00$ & $3398 \pm 5$ & $955.30 \pm 5.20$ & $0.14 \pm 0.00$ & $99928 \pm 5320$ \\
& 40 & $0.30 \pm 0.00$ & $3668 \pm 4$ & $40.20 \pm 0.19$ & $0.17 \pm 0.00$ & $38.20 \pm 1.03$ \\
\hline \multirow{3}{*}{$20 \%$-Gel } & 27 & $0.26 \pm 0.00$ & $9714 \pm 15$ & $3432.50 \pm 26.10$ & $0.27 \pm 0.00$ & $2.23 \mathrm{e} 6 \pm 1.33 \mathrm{e} 5$ \\
& 50 & $0.22 \pm 0.00$ & $12454 \pm 17$ & $6.09 \pm 0.05$ & $0.61 \pm 0.01$ & $4.58 \pm 0.22$ \\
& 70 & $0.28 \pm 0.00$ & $12780 \pm 23$ & $0.18 \pm 0.00$ & - & - \\
\hline
\end{tabular}

The relaxation modulus, $G(t)$ can be reasonably captured by a stretched exponential 
function, mathematically represented as $G(t)=G_{0} \exp \left(-\left(t / \tau_{S R}\right)^{\alpha}\right) .{ }^{4,6,7,18,60}$ Here, $G_{0}$ is zerotime shear modulus, $\tau_{S R}$ is the characteristic relaxation time, and $\alpha$ represents the stretch in the relaxation time spectrum. Note that $\alpha \rightarrow 1$ represents the Maxwell-type fluid with a single relaxation time. Although two exponent models can be used, ${ }^{35,61}$ the stretch exponential function is widely used to capture the stress-relaxation behavior of self-assembled gels with a broad distribution of relaxation time originated from their inhomogeneous microstructure. $^{4,6,7,18,60}$ Here, we fit this model to $G(t)$ over the time scale of 0.1 to $1800 \mathrm{~s}$. $G_{0}$, $\tau_{S R}$, and $\alpha$ have been treated as floating parameters and the results are shown in Table 2. Note that fitting the data up to $600 \mathrm{~s}$ does not display a significant variation in the fitting parameters.

An increase in $G_{0}$ and $\tau_{S R}$ with polymer concentration can be attributed to the higher number of PI-blocks participating as the load-bearing chains. ${ }^{8,47}$ A decrease in $\tau_{S R}$ at higher $T$ signifies increased chain mobility along with the increase in the solubility of PS-blocks in mineral oil. ${ }^{1,6}$ Both of these factors lead to a faster pullout of PS-blocks among the aggregates. The $\alpha$ values of both gels vary from $0.2-0.3$ indicating a distributed $\tau_{S R}$ due to the inhomogeneous microstructure of gels. For 20\%-Gel, $\alpha$ is slightly lower than that of 10\%-Gel suggesting a higher inhomogeneity of the microstructure. $4,6,7,18$

As shown in Figure 7A1-C1, the stress-relaxation and microstructure relaxation follow a similar trend. This suggests that the microstructure recovery time can also be represented in the form of stretched-exponential function as $\Omega(t)=\Omega_{0} \exp \left(-\left(t / \tau_{\Omega}\right)^{\alpha}\right)$, where $\Omega(t)$ is the time-dependent anisotropic factor, $\Omega_{0}$ signifies the anisotropy at $t \approx 0$, i.e., just after the application of strain, and $\tau_{\Omega}$ represents the characteristic recovery time of the microstructure, which is referred here as the microstructural relaxation time. Similar to $\tau_{S R}$, the $\tau_{\Omega}$ is also expected to have a broad distribution due to inhomogeneous microstructure, therefore, we have fixed the $\alpha$ values to those obtained from the fitting of stress-relaxation data. The fitted model captures the data reasonably well (Figure 7A1-C1), and the fitted parameters are shown in Table 2. 
Interestingly, for $10 \%-\mathrm{Gel}$ at $40{ }^{\circ} \mathrm{C}$ and for $20 \%$-Gel at $50{ }^{\circ} \mathrm{C}, \tau_{S R}$ and $\tau_{\Omega}$ are similar. For $70{ }^{\circ} \mathrm{C}$, due to fast relaxation time, sufficient microstructure data could not be collected. It signifies that the microstructural relaxation time is less than the sampling time in RheoSAXS $(3 \mathrm{~s})$. At $27^{\circ} \mathrm{C}, \tau_{\Omega}$ is approximately two orders of magnitude higher than the $\tau_{S R}$, which warrants further discussion.

After the application of step-strain, several processes can take place in a sample. The applied strain facilitates the end-block (PS-block) pullout, and such a process results in stress relaxation. The relaxation of PI-blocks also contributes to the stress-relaxation process. It has been shown that for the same endblock length, the stress relaxation is slower for longer midblock chains, particularly that with entanglements. ${ }^{2,7,8}$ The overall multimode relaxation process has the characteristic time scale of $\tau_{S R}$. The pulled-out endblocks can reassociate to an existing aggregate, or multiple of the endblocks associate to form new aggregates. Both chain pullout and chain exchange have to be completed to observe the microstructural relaxation in the scattering profile. It appears that the process is much slower than the stress-relaxation process at the room temperature. We attribute this to the slow endblock diffusion process through the crowded environment retarded by the high chain density and entanglements of PI-blocks. ${ }^{6,7,25}$ This is further evident from 20\%-Gel sample in which higher polymer concentration leads to the larger difference between $\tau_{S R}$ and $\tau_{\Omega}$. In addition to these processes, various other mechanisms like corona screening, double activation, and walking diffusion are also shown to affect the relaxation dynamics in gels. ${ }^{25,62,63}$ Note that microstructure recovery is faster during the oscillation experiments facilitated by the applied strain.

\section{Concluding Remarks}

We have related the microstructure and rheology results for 10 and 20 wt\% of PS-PI-PS triblock copolymer gels in mineral oil during temperature-dependent gelation, subjected to 
large amplitude oscillatory strain, and during the stress-relaxation process. During gelation, loosely bounded aggregates are observed at higher temperatures than $T_{g e l}$ obtained from shear-rheology experiments. The microstructure evolved with decreasing temperature, and at room temperature, the microstructure evolution is complete. Correspondingly, $G^{\prime}$ reaches a plateau. With the application of strain, the gel microstructure orients in the stretching direction. This results in the transformation of the circular scattering pattern in the isotropic state to an oriented elliptical scattering pattern. However, in an oscillatory strain cycle with high strain amplitude, a split in scattering pattern has been observed with increasing intracycle strain, $\gamma$, displaying both ellipse and circle patterns. This has been attributed to chain pullout caused by strain localization. A polydisperse core hard-sphere model can capture the scattering data both at the static and under loading conditions. The estimated results are comparable to that estimated using affine deformation. In the stress-relaxation experiments, the microstructure relaxes faster at higher temperatures. The characteristic stress-relaxation time and microstructural relaxation time, estimated using a stretched-exponential model, are similar at higher temperatures but deviate significantly at room temperature. This has been attributed to the complex multimode relaxation process including PI-block relaxation, PSblock pull-out and reassociation process. Further understanding of the relaxation behavior needs experimental investigations such as dielectric measurement, ${ }^{64}$ and SANS with appro-

priate contrast matching, ${ }^{25}$ which can capture the A-block pullout and B-block relaxation process. Future experiments will also involve the investigation of microstructural relaxation process by varying the A- or B-block lengths.

\section{Acknowledgment}

This work was supported by the National Science Foundation [DMR-1352572, DMR-2004501]. RheoSAXS experiments were conducted at the DuPont-Northwestern-Dow Collaborative Access Team (DND-CAT) Synchrotron Research Center located at Sector 5 of the Advanced 
Photon Source. This research used resources of the Advanced Photon Source, a U.S. Department of Energy (DOE) Office of Science User Facility operated for the DOE Office of Science by Argonne National Laboratory under Contract No. DE-AC02-06CH11357. We acknowledge Dr. Steven Weigand for assisting in the RheoSAXS experiments.

\section{References}

(1) Laurer, J. H.; Khan, S. A.; Spontak, R. J.; Satkowski, M. M.; Grothaus, J. T.; Smith, S. D.; Lin, J. S. Morphology and Rheology of SIS and SEPS Triblock Copolymers in the Presence of a Midblock-Selective Solvent. Langmuir 1999, 15, 7947-7955.

(2) Vega, D. A.; Sebastian, J. M.; Loo, Y. L.; Register, R. A. Phase Behavior and Viscoelastic Properties of Entangled Block Copolymer Gels. J. Polym. Sci. Part B Polym. Phys. 2001, 39, 2183-2197.

(3) Drzal, P. L.; Shull, K. R. Origins of Mechanical Strength and Elasticity in Thermally Reversible, Acrylic Triblock Copolymer Gels. Macromolecules 2003, 36, 2000-2008.

(4) Seitz, M. E.; Burghardt, W. R.; Faber, K. T.; Shull, K. R. Self-Assembly and Stress Relaxation in Acrylic Triblock Copolymer Gels. Macromolecules 2007, 40, 1218-1226.

(5) Seitz, M. E.; Rottsolk, R. L.; Shull, K. R. Effect of Homopolymer Solubilization on Triblock Gel Structure and Mechanical Response. Journal of Polymer Science Part B: Polymer Physics 2010, 48, 1395-1408.

(6) Mishra, S.; Badani Prado, R. M.; Lacy, T. E.; Kundu, S. Investigation of Failure Behavior of a Thermoplastic Elastomer Gel. Soft Matter 2018, 14, 7958-7969.

(7) Mishra, S.; Badani Prado, R. M.; Zhang, S.; Lacy, T. E.; Gu, X.; Kundu, S. Mechanical Properties and Failure Behavior of Physically Assembled Triblock Copolymer Gels with 
Varying Midblock Length. Journal of Polymer Science Part B: Polymer Physics 2019, polb.24860.

(8) Mishra, S.; Badani Prado, R. M.; Kundu, S. Concentration-Dependent Mechanical Behavior of Physically Assembled Triblock Copolymer Gels. ACS Applied Polymer Materials 2020, 2, 5388-5397.

(9) Madsen, J.; Armes, S. P.; Bertal, K.; Lomas, H.; MacNeil, S.; Lewis, A. L. Biocompatible Wound Dressings based on Chemically Degradable Triblock Copolymer Hydrogels. Biomacromolecules 2008, 9, 2265-2275.

(10) Censi, R.; Vermonden, T.; van Steenbergen, M. J.; Deschout, H.; Braeckmans, K.; De Smedt, S. C.; van Nostrum, C. F.; di Martino, P.; Hennink, W. E. Photopolymerized Thermosensitive Hydrogels for Tailorable Diffusion-Controlled Protein Delivery. Journal of Controlled Release 2009, 140, 230-236.

(11) Vermonden, T.; Jena, S. S.; Barriet, D.; Censi, R.; Van Der Gucht, J.; Hennink, W. E.; Siegel, R. A. Macromolecular diffusion in self-assembling biodegradable thermosensitive hydrogels. Macromolecules 2010, 43, 782-789.

(12) Yu, L.; Zhang, Z.; Zhang, H.; Ding, J. Biodegradability and biocompatibility of thermoreversible hydrogels formed from mixing a sol and a precipitate of block copolymers in water. Biomacromolecules 2010, 11, 2169-2178.

(13) Mrozek, R. A.; Leighliter, B.; Gold, C. S.; Beringer, I. R.; Yu, J. H.; Vanlandingham, M. R.; Moy, P.; Foster, M. H.; Lenhart, J. L. The Relationship between Mechanical Properties and Ballistic Penetration Depth in a Viscoelastic Gel. J. Mech. Behav. Biomed. Mater. 2015, 44, 109-120.

(14) Sudarsan, A. P.; Wang, J.; Ugaz, V. M. Thermoplastic Elastomer Gels: An Advanced Substrate for Microfluidic Chemical Analysis Systems. Anal. Chem. 2005, 77, 51675173. 
(15) Trivedi, D.; Rahn, C. D.; Kier, W. M.; Walker, I. D. Soft robotics: Biological inspiration, state of the art, and future research. Applied Bionics and Biomechanics 2008, 5, 99117.

(16) Badani Prado, R. M.; Mishra, S.; Morgan, B.; Wijayapala, R.; Hashemnejad, S. M.; Kundu, S. Achieving High-Speed Retraction in Stretchable Hydrogels. ACS applied materials $\&$ interfaces 2020, 12, 40719-40727.

(17) Wijayapala, R.; Mishra, S.; Elmore, B.; Freeman, C.; Kundu, S. Synthesis and Characterization of Crosslinked Polymers from Cottonseed Oil. Journal of Applied Polymer Science 2019, 136, 47655 .

(18) Zabet, M.; Mishra, S.; Kundu, S. Effect of Graphene on the Self-Assembly and Rheological Behavior of a Triblock Copolymer Gel. Rsc. Adv. 2015, 5, 83936-83944.

(19) Bras, R. E.; Shull, K. R. Self-Consistent Field theory of Gelation in Triblock Copolymer Solutions. Macromolecules 2009, 42, 8513-8520.

(20) Chantawansri, T. L.; Sirk, T. W.; Sliozberg, Y. R. Entangled Triblock Copolymer Gel: Morphological and Mechanical Properties. J. Chem. Phys. 2013, 138, 1-11.

(21) Chantawansri, T. L.; Sirk, T. W.; Mrozek, R. A.; Lenhart, J. L.; Kröger, M.; Sliozberg, Y. R. The Effect of Polymer Chain Length on the Mechanical Properties of Triblock Copolymer Gels. Chem. Phys. Lett. 2014, 612, 157-161.

(22) Zabet, M.; Mishra, S.; Boy, R.; Walters, K. B.; Naskar, A. K.; Kundu, S. TemperatureDependent Self-Assembly and Rheological Behavior of a Thermoreversible PMMAPnBA-PMMA Triblock Copolymer Gel. J. Polym. Sci. Part B Polym. Phys. 2017, 55, $877-887$.

(23) Inomata, K.; Nakanishi, D.; Banno, A.; Nakanishi, E.; Abe, Y.; Kurihara, R.; Fuji- 
moto, K.; Nose, T. Association and Physical Gelation of ABA Triblock Copolymer in Selective Solvent. Polymer (Guildf). 2003, 44, 5303-5310.

(24) Erk, K. A.; Henderson, K. J.; Shull, K. R. Strain Stiffening in Synthetic and Biopolymer Networks. Biomacromolecules 2010, 11, 1358-1363.

(25) Peters, A. J.; Lodge, T. P. Comparison of Gel Relaxation Times and End-Block Pullout Times in ABA Triblock Copolymer Networks. Macromolecules 2016, 49, 7340-7349.

(26) Mortensen, K. Three-dimensional Crystallographic Determination of the BodyCentered-Cubic Morphologies of Shear-Aligned Block Copolymer Systems. Journal of Polymer Science Part B: Polymer Physics 2004, 42, 3095-3101.

(27) Gopinadhan, M.; Majewski, P. W.; Choo, Y.; Osuji, C. O. Order-Disorder Transition and Alignment Dynamics of a Block Copolymer Under High Magnetic Fields by In Situ X-Ray Scattering. Physical Review Letters 2013, 110, 078301.

(28) Wiegart, L.; Doerk, G. S.; Fukuto, M.; Lee, S.; Li, R.; Marom, G.; Noack, M. M.; Osuji, C. O.; Rafailovich, M. H.; Sethian, J. A.; Shmueli, Y.; Torres Arango, M.; Toth, K.; Yager, K. G.; Pindak, R. Instrumentation for In situ/Operando X-ray Scattering Studies of Polymer Additive Manufacturing Processes. Synchrotron Radiation News 2019, 32, 20-27.

(29) Chu, B.; Hsiao, B. S. Small-Angle X-ray Scattering of Polymers. Chemical Reviews 2001, 101, 1727-1762.

(30) Caputo, F. E.; Burghardt, W. R. Real-time 1-2 Plane SAXS Measurements of Molecular Orientation in Sheared Liquid Crystalline Polymers. Macromolecules 2001, 34, 66846694.

(31) Stribeck, N.; Nöchel, U.; Funari, S. S.; Schubert, T. Tensile tests of polypropylene 
monitored by SAXS. Comparing the stretch-hold technique to the dynamic technique. Journal of Polymer Science Part B: Polymer Physics 2008, 46, 721-726.

(32) Yu, J. M.; Dubois, P.; Teyssié, P.; Jérôme, R. Syndiotactic poly(methyl methacrylate) (sPMMA)-polybutadiene (PBD)-sPMMA Triblock Copolymers: Synthesis, Morphology, and Mechanical Properties. Macromolecules 1996, 29, 6090-6099.

(33) Maranzano, B. J.; Wagner, N. J. Flow-small Angle Neutron Scattering Measurements of Colloidal Dispersion Microstructure Evolution through the Shear Thickening Transition. The Journal of Chemical Physics 2002, 117, 10291-10302.

(34) Murphy, R. P.; Riedel, Z. W.; Nakatani, M. A.; Salipante, P. F.; Weston, J. S.; Hudson, S. D.; Weigandt, K. M. Capillary RheoSANS: Measuring the Rheology and Nanostructure of Complex Fluids at High Shear Rates. Soft Matter 2020, 16, 6285-6293.

(35) Sing, M. K.; Glassman, M. J.; Vronay-Ruggles, X. T.; Burghardt, W. R.; Olsen, B. D. Structure and Rheology of Dual-Associative Protein Hydrogels under Nonlinear Shear Flow. Soft Matter 2017, 13, 8511-8524.

(36) Liberatore, M. W.; Nettesheim, F.; Wagner, N. J.; Porcar, L. Spatially Resolved SmallAngle Neutron Scattering in the 1-2 Plane: a Study of Shear-induced Phase-Separating Wormlike Micelles. Physical Review E 2006, 73, 020504.

(37) Liberatore, M. W.; Nettesheim, F.; Vasquez, P. A.; Helgeson, M. E.; Wagner, N. J.; Kaler, E. W.; Cook, L. P.; Porcar, L.; Hu, Y. T. Microstructure and Shear Rheology of Entangled Wormlike Micelles in Solution. Journal of Rheology 2009, 53, 441-458.

(38) Seitz, M. E.; Martina, D.; Baumberger, T.; Krishnan, V. R.; Hui, C. Y.; Shull, K. R. Fracture and Large Strain Behavior of Self-Assembled Triblock Copolymer Gels. Soft Matter 2009, 5, 447-456. 
(39) Weigandt, K. M.; Porcar, L.; Pozzo, D. C. In Situ Neutron Scattering Study of Structural Transitions in Fibrin Networks under Shear Deformation. Soft Matter 2011, 7, 9992.

(40) Caputo, F. E.; Burghardt, W. R.; Krishnan, K.; Bates, F. S.; Lodge, T. P. Time-resolved Small-angle X-ray Scattering Measurements of a Polymer Bicontinuous Microemulsion Structure Factor under Shear. Physical Review E - Statistical Physics, Plasmas, Fluids, and Related Interdisciplinary Topics 2002, 66, 18.

(41) Ilavsky, J. Nika: Software for Two-dimensional Data Reduction. Journal of Applied Crystallography 2012, 45, 324-328.

(42) Kline, S. R. Reduction and Analysis of SANS and USANS Data using Igor Pro. J. Appl. Crystallogr. 2006, 39, 895-900.

(43) Winter, H. H.; Chambon, F. Analysis of Linear Viscoelasticity of a Crosslinking Polymer at the Gel Point. J. Rheol. (N. Y. N. Y). 1986, 30, 367-382.

(44) Soenen, H.; Berghmans, H.; Winter, H.; Overbergh, N. Ordering and structure formation in triblock copolymer solutions. Part I. Rheological observations. Polymer 1997, $38,5653-5660$.

(45) Yu, J. M.; Dubois, P.; Teyssié, P.; Jérôme, R.; Blacher, S.; Brouers, F.; L’Homme, G. Triblock Copolymer based Thermoreversible Gels. 2. Analysis of the Sol-Gel Transition. Macromolecules 1996, 29, 5384-5391.

(46) Sato, T.; Watanabe, H.; Osaki, K. Thermoreversible Physical Gelation of Block Copolymers in a Selective Solvent. Macromolecules 2000, 33, 1686-1691.

(47) Ye, Y. N.; Cui, K.; Indei, T.; Nakajima, T.; Hourdet, D.; Kurokawa, T.; Gong, J. P. Relaxation Dynamics and Underlying Mechanism of a Thermally Reversible Gel from Symmetric Triblock Copolymer. Macromolecules 2019, 52, 8651-8661. 
(48) Mischenko, N.; Reynders, K.; Koch, M. H. J.; Mortensen, K.; Pedersen, J. S.; Fontaine, F.; Graulus, R.; Reynaers, H. Small-Angle X-Ray and Neutron Scattering from Bulk and Oriented Triblock Copolymer Gels. Macromolecules 1995, 28, 20542062.

(49) Rubinstein, M.; Colby, R. Polymer Physics; OUP Oxford, 2003.

(50) Treloar, L. R. G. The Physics of Rubber Elasticity; Oxford University Press, USA, 1975.

(51) Erk, K. A.; Shull, K. R. Rate-Dependent Stiffening and Strain Localization in Physically Associating Solutions. Macromolecules 2011, 44, 932-939.

(52) Erk, K. A.; Martin, J. D.; Hu, Y. T.; Shull, K. R. Extreme Strain Localization and Sliding Friction in Physically Associating Polymer Gels. Langmuir 2012, 28, 44724478.

(53) Hashemnejad, S. M.; Kundu, S. Nonlinear Elasticity and Cavitation of a Triblock Copolymer Gel. Soft Matter 2015, 11, 4315-4325.

(54) Ewoldt, R. H.; Hosoi, a. E.; McKinley, G. H. New Measures for Characterizing Nonlinear Viscoelasticity in Large Amplitude Oscillatory Shear. Journal of Rheology 2008, 52, 1427.

(55) Hyun, K.; Wilhelm, M.; Klein, C. O.; Cho, K. S.; Nam, J. G.; Ahn, K. H.; Lee, S. J.; Ewoldt, R. H.; McKinley, G. H. A Review of Nonlinear Oscillatory Shear Tests: Analysis and Application of Large Amplitude Oscillatory Shear (LAOS). Progress in Polymer Science 2011, 36, 1697-1753.

(56) Shan, L.; He, H.; Wagner, N. J.; Li, Z. Nonlinear Rheological Behavior of Bitumen Under LAOS Stress. Journal of Rheology 2018, 62, 975-989.

(57) Kundu, S.; Hashemnejad, S. M.; Zabet, M.; Mishra, S. Gels and Other Soft Amorphous Solids; American Chemical Society: Washington, DC, 2018; Chapter 9, pp 157-197. 
(58) Larson, R. G. Arrested Tumbling in Shearing Flows of Liquid Crystal Polymers. Macromolecules 1990, 23, 3983-3992.

(59) Hongladarom, K.; Burghardt, W. R. Measurement of the Full Refractive Index Tensor in Sheared Liquid Crystalline Polymer Solutions. Macromolecules 1994, 27, 483-489.

(60) Erk, K. A.; Douglas, J. F. Stretched Exponential Stress Relaxation in a Thermally Reversible, Physically Associating Block Copolymer Solution. MRS Proc. 2012, 1418.

(61) Calabrese, M. A.; Wagner, N. J. Detecting Branching in Wormlike Micelles via Dynamic Scattering Methods. ACS Macro Letters 2018, 7, 614-618.

(62) Yokoyama, H.; Kramer, E. J.; Fredrickson, G. H. Simulation of Diffusion of Asymmetric Diblock and Triblock Copolymers in a Spherical Domain Structure. Macromolecules 2000, 33, 2249-2257.

(63) Yokoyama, H.; Kramer, E. J. Diffusion of Triblock Copolymers in a Spherical Domain Structure. Macromolecules 2000, 33, 954-959.

(64) Watanabe, H.; Sato, T.; Osaki, K. Concentration Dependence of Loop Fraction in Styrene-Isoprene-Styrene Triblock Copolymer Solutions and Corresponding Changes in Equilibrium Elasticity. Macromolecules 2000, 33, 2545-2550. 


\section{Supplementary Information ${ }^{\dagger}$ :}

\section{Capturing the Transient Microstructure of a}

\section{Physically Assembled Gel Subjected to \\ Temperature and Large Deformation}

Rosa Maria Badani Prado, ${ }^{\dagger}$ Satish Mishra, ${ }^{\dagger}$ Wesley R. Burghardt, ${ }^{\ddagger}$ and Santanu

$$
\text { Kundu*,† }
$$

†Dave C. Swalm School of Chemical Engineering, Mississippi State University, MS State, $M S$ 39762.

$\ddagger$ Department of Chemical Engineering, Northwestern University, Evanston, Illinois 60208

$$
\text { E-mail: santanukundu@che.msstate.edu }
$$

Table S1: Gel nomenclature, polymer concentration in wt\%, polymer volume fraction $(\phi)$, and PS and PI content in the gels (wt\%).

\begin{tabular}{ccccc}
\hline Sample & wt\% & $\phi(\mathrm{v} / \mathrm{v})$ & PS (wt\%) & PI (wt\%) \\
\hline 10\%-Gel & 10 & 0.089 & 2.9 & 7.1 \\
$20 \%$-Gel & 20 & 0.181 & 5.8 & 14.2 \\
\hline
\end{tabular}




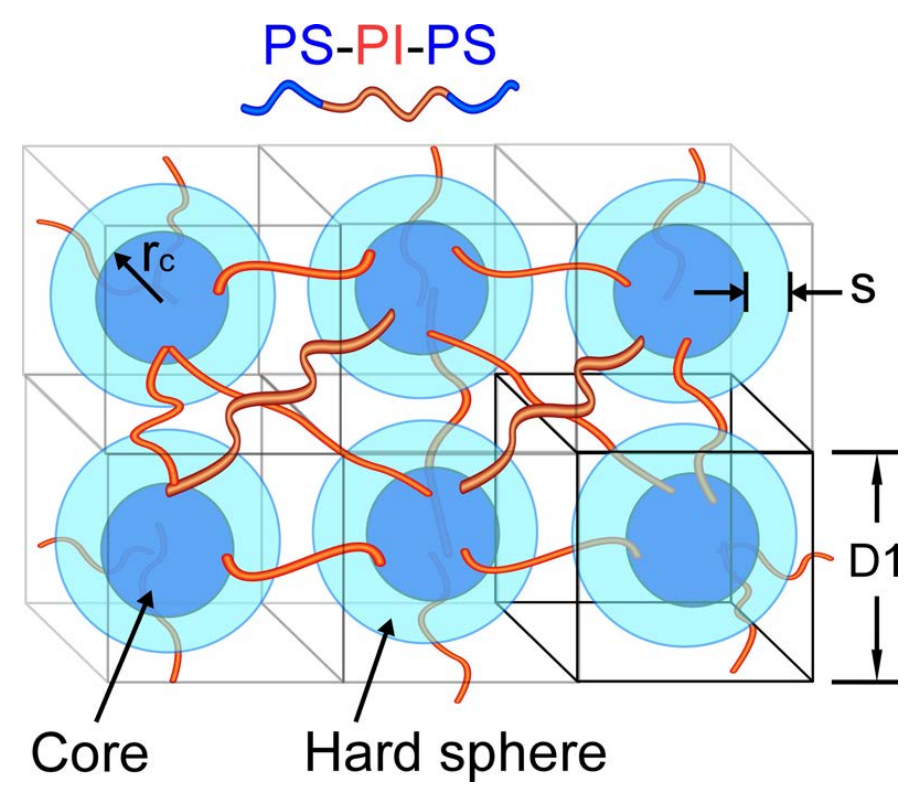

Figure S1: Schematic representing gel structure. 3D crosslinked network formed by poly(styrene)-poly(isoprene)-poly(styrene) [PS-PI-PS] triblock copolymer in mineral oil, where the PS-blocks form aggregates bridged by PI-blocks. The PS-aggregates of core radius $r_{c}$ are surrounded by a fictitious hard sphere of thickness $s$ contained in a cube with dimension of $D_{1}$.
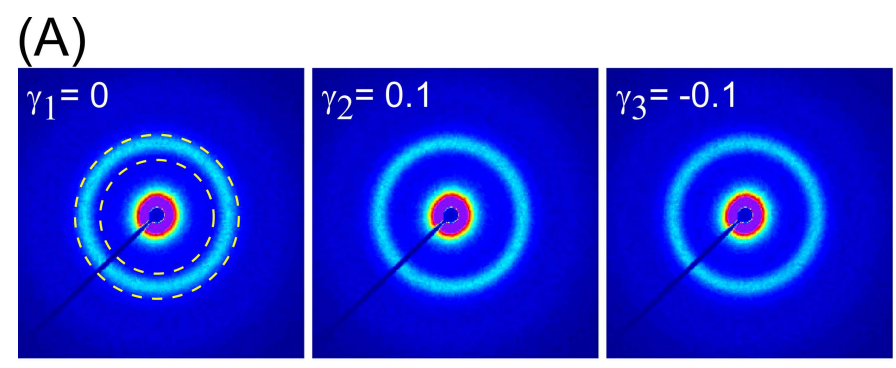

(B)
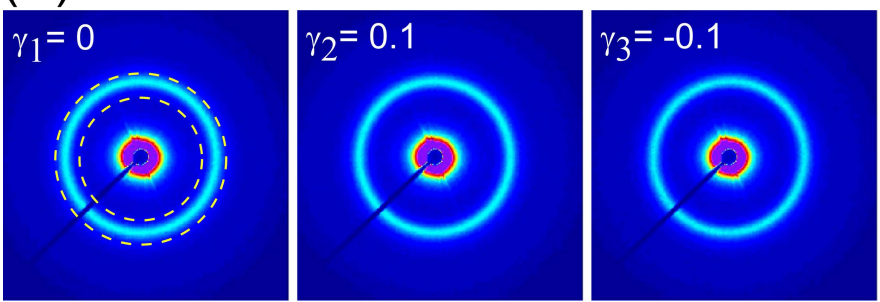

Figure S2: 2D-scattering patterns for gels at $27{ }^{\circ} \mathrm{C}$ during a strain cycle with $\gamma_{a m p}$ of 0.1 . 2D-scattering patterns are shown for $\gamma$ (intracycle strain) equal to $0,0.1$, and -0.1 for the (A) $10 \%$-Gel and (B) 20\%-Gel. The dashed lines in the $\gamma_{1}$ images indicate the analyzed $q$-range. 
(A)

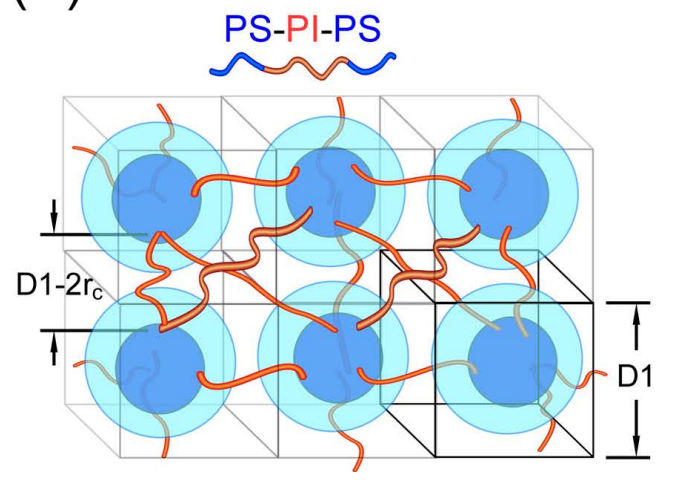

(B)

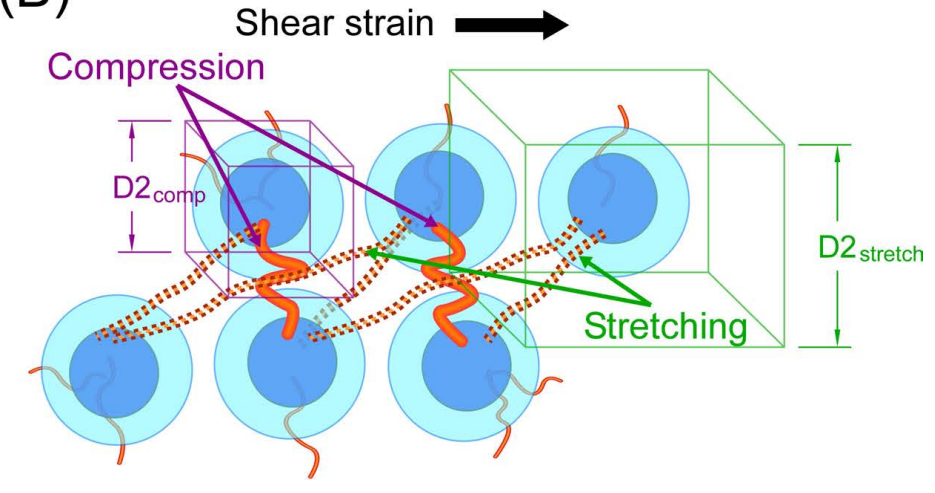

Figure S3: Gel structure at the static condition and after the application of a shear strain. (A) Gel structure formed by PS-aggregates surrounded by a fictitious hard sphere contained in a cube with dimension $D_{1}$ at the static condition. (B) The change of structure after a shear strain is applied, displaying compressed, and stretched chains. The corresponding cube has dimension of $D_{2}$

(A)

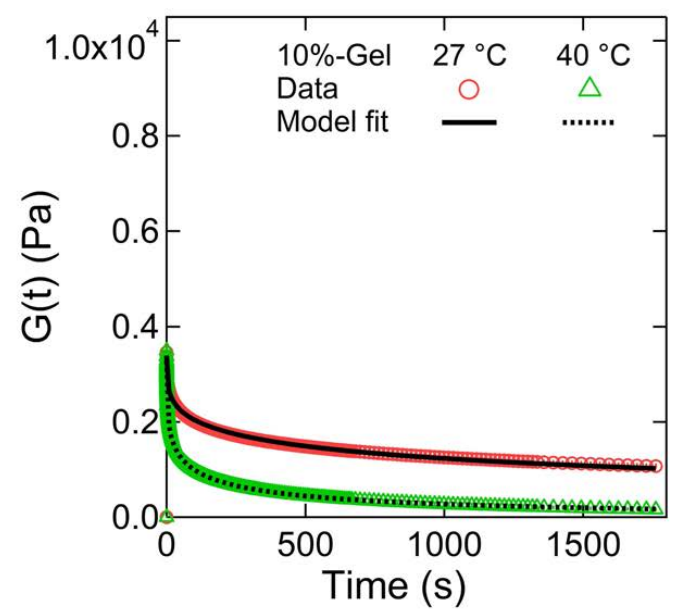

(B)

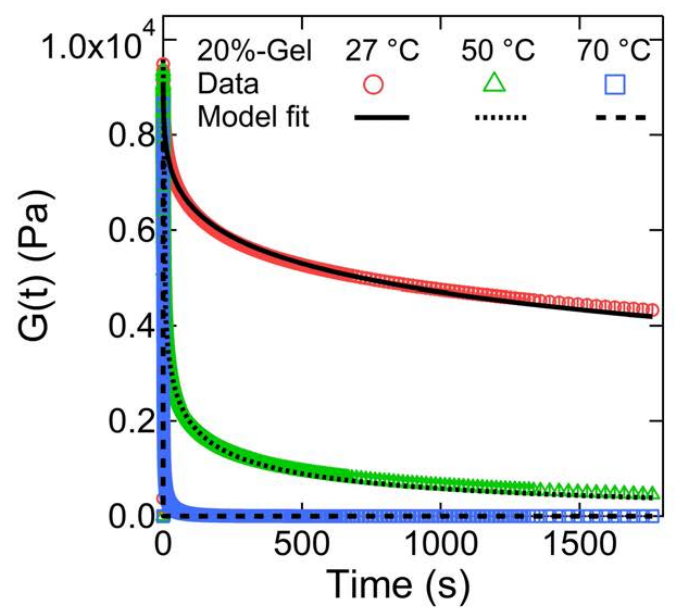

Figure S4: Stress relaxation for 10\%- and 20\%-Gels over $1800 \mathrm{~s}$ at different temperatures obtained from shear rheology experiments. Time-dependent modulus $(G(t))$ over time with an applied step strain $\left(\gamma_{\text {step }}\right)$ of 0.3 , and fitted with a stretched exponential function for the (A) $10 \%$-Gel at 27 and $40{ }^{\circ} \mathrm{C}$, and the (B) $20 \%$-Gel at 27,50 , and $70{ }^{\circ} \mathrm{C}$. 


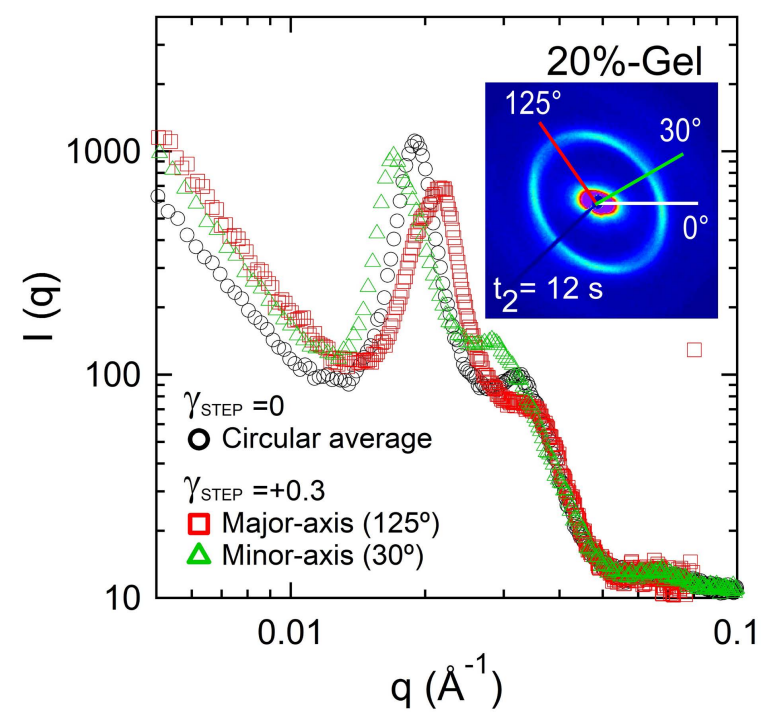

Figure S5: $I(q)-q$ data corresponding to $t_{2}$ obtained from RheoSAXS experiments for $20 \%$ Gel at $50{ }^{\circ} \mathrm{C}$. A step strain $\gamma_{s t e p}=0.3$ was applied and the gel was allowed to relax. Here, the sector averages along major- and minor-axis $\left(\beta=125^{\circ}\right.$ and $\left.30^{\circ}\right)$ are compared with the circular average data at the static condition $\left(\gamma_{s t e p}=0\right)$. The inset represents the oriented 2 D-scattering pattern along with the azimuthal angles $(\beta)$ corresponding to the major- and minor-axes. 
(A1)

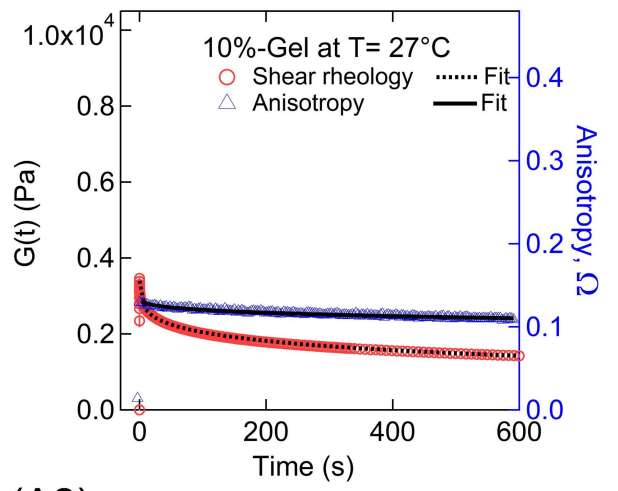

(A2)
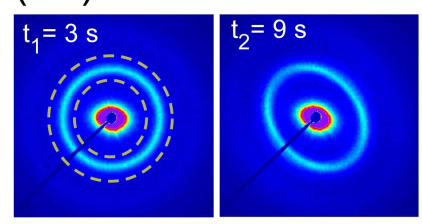

(A3)

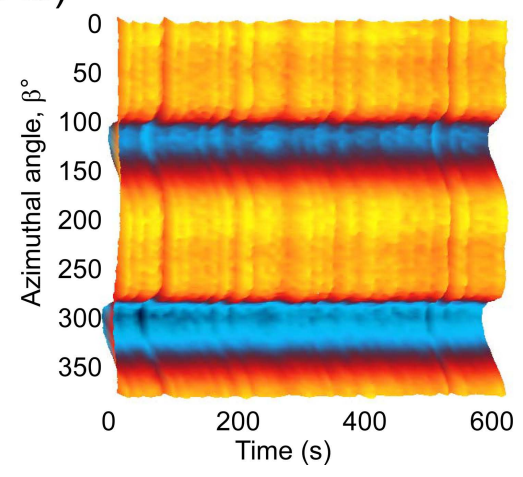

(B1)

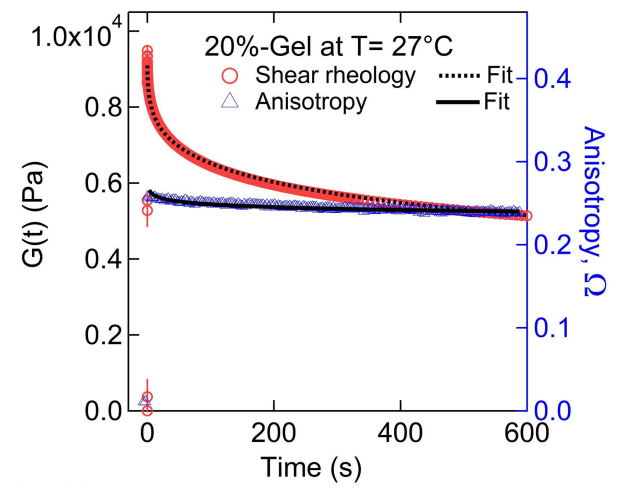

(B2)
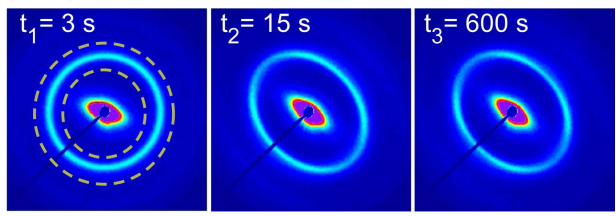

(B3)

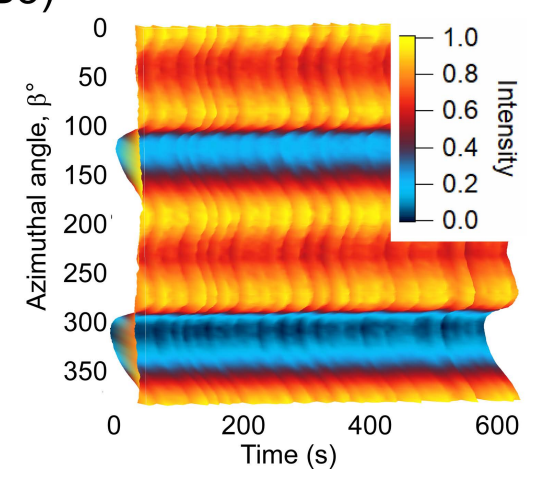

Figure S6: Results for the stress relaxation experiments at $27{ }^{\circ} \mathrm{C}$, azimuthal plots, and 2D-scattering patterns for gels. Time-dependent modulus $(G(t))$ fitted with stretched exponential function, 2D-scattering patterns at different time points, and stacked azimuthal plots over time are shown for the (A) $10 \%$-Gel and (B) $20 \%$-Gel. Here, $t_{2}$ is just after the strain is applied. The dashed lines in the $t_{1}$-images indicate the analyzed $q$-range for each case. 


\section{Model description}

Model fit for the gels at static conditions: We have characterized the gel microstructure using a polydispersed core hard-sphere model for the gels at static conditions. According to this model, the PS-aggregates can be viewed as cores having a Gaussian distribution in radius. The cores have a mean radius $r_{c}$ with polydispersity $\sigma / r_{c}$. The cores are surrounded by fictitious hard spheres, concentric to the cores, with thickness $s_{1}$ estimating the radius of hard spheres as $r_{c}+s_{1}$. The hard spheres occupy a volume fraction $\phi_{1}$ in the gel. The model is a combination of a spherical form factor $(P(q))$ multiplied with hard-sphere structure factor $(S(q))$, mathematically represented as $I(q) \propto P(q) \times S_{1}(q)$. The inter-aggregate distance, approximately representing the end-to-end distance of a PI-block in the gel can also be calculated as $D_{1}=\left(4 \pi R^{3} /\left(3 \psi_{1}\right)\right)^{1 / 3},{ }^{1}$ where $R=r_{c}+s_{1}$ is the radius of the hard-sphere. The above model is also used for the cases in which the gel is under deformation but the $2 \mathrm{D}$ scattering pattern does not display a split.

Model fit for the gels at stretched conditions: For the gels at $\gamma= \pm 1$, a modified polydispersed core hard-sphere model was used for the fitting. The model is justified as follows. At these $\gamma$ values, we observed a circular pattern and an elliptical pattern. The circular pattern resembling the scattering pattern at the static condition. The oriented microstructure displays an elliptical pattern. The $I(q)-q$ curve displays two length scales viz. (1) along the minor-axis, displaying the increase in the inter-aggregate distance as a result of applied $\gamma$, and (2) along the major-axis, signifying a decrease in the inter-aggregate distance or compression as a consequence of stretching in the orthogonal direction (minoraxis). The state of the hard spheres in case- 1 and -2 can be referred to as stretched and compressed, respectively, when compared with those at static conditions. Therefore, at a particular $\gamma$, three inter-aggregate distances can be envisaged - unperturbed, increased, and decreased. Along with the minor-axis sector, the $I(q)-q$ data signifies two arrangements of hard spheres- unperturbed and stretched. Similarly, along the major-axis, the data represents unperturbed and compressed hard spheres. Note that our data indicates an intact form factor 
peak, meaning that the aggregates do not change the size and shape during deformation. Since the value of the hard-sphere radius $(R)$ signifies the inter-aggregate distance, the changed inter-aggregate distance can be conveniently represented by adding a hard-sphere in the model while keeping the form factor the same. We represent the modified polydispersed hard-sphere model as $I(q) \propto P(q) \times\left(S_{1}(q)+S_{2}(q)\right)$, where $S_{2}(q)$ is the structure factor related to the new hard-sphere to capture the characteristics of displaced hard spheres.

Mathematical expression: Here, the structure factor is calculated based on PercusYevick closure as

$$
S(q)=\frac{1}{1+25 \psi G(A) / A}
$$

where $A=4 \pi q R, R$ is the radius of hard sphere, and $\psi$ is the hard-sphere volume fraction.

$$
\begin{aligned}
G(A)= & \alpha(\sin A-A \cos A)+\beta / A^{3}\left(2 A \sin A+\left(2-A^{2}\right) \cos A-2\right)+ \\
& \gamma\left(-A^{4} \cos A+4\left(\left(3 A^{2}-6\right) \cos A+\left(A^{3}-6 A\right) \sin A+6\right)\right) / A^{5}
\end{aligned}
$$

and

$$
\begin{gathered}
\alpha=\frac{(1+2 \psi)^{2}}{(1-\psi)^{4}} \\
\beta=-6 \psi\left(1+\frac{\psi}{2}\right)^{2} /(1-\psi)^{2} \\
\gamma=\frac{\psi \alpha}{2}
\end{gathered}
$$

Form factor of sphere can be mathematically expressed as:

$$
P_{1}(q, r)=\left(\frac{4 \pi r_{c}^{3}}{3}\right)^{2}\left(\left(\frac{3}{q r_{c}}\right)^{3}\left(\sin \left(q r_{c}\right)-q r_{c} \cos \left(q r_{c}\right)\right)\right)^{2}
$$

The polydispersity is $\sigma / r_{c}$, where $\sigma$ is the variance of distribution in aggregate radius 
$r_{c}$. And the average form factor is:

$$
\left\langle P\left(q, r_{c}, \sigma\right)\right\rangle=\int_{0}^{\infty} \frac{1}{\sigma(2 \pi)^{1 / 2}} \exp \left(-\frac{\left(r-r_{c}\right)^{2}}{2 \sigma^{2}}\right) F(q, r) d r
$$

\section{References}

(1) Seitz, M. E.; Burghardt, W. R.; Faber, K. T.; Shull, K. R. Self-Assembly and Stress Relaxation in Acrylic Triblock Copolymer Gels. Macromolecules 2007, 40, 1218-1226. 